\title{
Capacitively coupled radio-frequency discharges in nitrogen at low pressure
}

\author{
L L Alves ${ }^{1}$, L Marques $^{1,2}$, C D Pintassilgo ${ }^{1,3}$, G Wattieaux $^{4}$, Et \\ Es-sebbar $^{5,6}$, J Berndt ${ }^{4}$, E Kovačević $^{4}, \mathbf{N}$ Carrasco $^{5}$, L \\ Boufendi $^{4}$ and G Cernogora ${ }^{5}$ \\ 1 Instituto de Plasmas e Fusão Nuclear, Instituto Superior Técnico, Universidade \\ Técnica de Lisboa, 1049-001 Lisboa, Portugal \\ 2 Centro de Física da Universidade do Minho, Universidade do Minho, 4710-057, \\ Braga, Portugal \\ 3 Departamento Engenharia Física, Faculdade Engenharia, Universidade do Porto, R. \\ Dr. Roberto Frias, 4200-465 Porto, Portugal \\ ${ }^{4}$ GREMI Université d'Orléans BP 6744 - 45067 Orléans France \\ ${ }^{5}$ LATMOS-UVSQ-CNRS 11 Bd d'Alembert 78280 Guyancourt, France \\ ${ }^{6}$ Clean Combustion Research Center, King Abdullah University of Science and \\ Technology (KAUST), Thuwal 23955, Saudi Arabia \\ E-mail: llalves@ist.utl.pt
}

\begin{abstract}
This paper uses experiments and modelling to study capacitively coupled radio-frequency discharges in pure nitrogen, at $13.56 \mathrm{MHz}$ frequency, $0.1-1 \mathrm{mbar}$ pressures and $2-30 \mathrm{~W}$ coupled powers. Experiments performed on two similar (not twin) setups, existing in the LATMOS and the GREMI laboratories, include electrical and optical emission spectroscopy (OES) measurements. Electrical measurements give the rf-applied and the dc-self-bias voltages, the effective power coupled to the plasma and the average electron density. OES diagnostics measure the intensities of radiative transitions with the nitrogen second-positive and first-negative systems, and with the $811.5 \mathrm{~nm}$ atomic line of argon (present as an actinometer). Simulations use a hybrid code that couples a two-dimensional time-dependent fluid module, describing the dynamics of the charged particles (electrons and positive ions $\mathrm{N}_{2}^{+}$and $\mathrm{N}_{4}^{+}$), and a zero-dimensional kinetic module, describing the production and destruction of nitrogen (atomic and molecular) neutral species. The coupling between these modules adopts the local mean energy approximation to define space-time dependent electron parameters for the fluid module and to work-out space-time average rates for the kinetic module. The model gives general good predictions for the self-bias voltage and for the intensities of radiative transitions (both average and spatially-resolved), underestimating the electron density by a factor of $3-4$.
\end{abstract}

PACS numbers: 52.80.Pi, 52.70.-m, 52.65.Ww, 52.20.-j

Submitted to: Plasma Sources Sci. Technol. 


\section{Introduction}

Radio-frequency (rf) discharge plasmas produced in nitrogen and nitrogen mixtures are becoming increasingly popular, because they exhibit a high chemical reactivity leading to the production of active radicals and ions, even at low pressure and ambient temperature. These plasmas can be produced using various rf-excitation techniques (dielectric barrier, inductive coupling and capacitive coupling), and they are frequently used for the processing, modification and functionalization of different kinds of materials, such as fullerenes $[1,2]$, nanotubes and nanofibres $[3,4,5]$, polymers $[6,7,8,9]$ and textiles $[10,11]$. Nitrogen-containing rf-plasmas are also used to grow GaN thin films $[12,13]$ and in the production of hydrogenated carbon nitride materials, from thin films $[14,15,16,17,18,19,20,21]$ to nanoparticles [22, 23]. Nitrogen-doped carbonaceous materials can exhibit numerous interesting properties, such as extreme hardness [24] or increased biocompatibility [25, 26, 27, 28]. The use of nitrogen/methane mixtures to produce capacitively coupled radio-frequency (ccrf) dusty plasmas (with $\mathrm{CH}_{4}$ concentrations below 10\%) is another very active research field. The purpose of these investigations is the simulation in laboratory of the chemistry of Titan's atmosphere, including the synthesis of analogues of its organic solid aerosols [29]. These analogues (named tholins) are probably produced by photochemistry processes induced by the solar radiation upon charged particles from Saturn's magnetosphere. To ensure that the dust particles produced in a laboratory plasma environment are representatives of Titan's aerosols, one needs to characterize the plasma source controlling the reactivity of the $\mathrm{N}_{2}-\mathrm{CH}_{4}$ mixture, comparing it with the photon source that provides the energy required to grow the solid particles of Titan. Several experimental works have been carried out with this intention, involving a wide number of different $\mathrm{N}_{2}-\mathrm{CH}_{4}$ plasmas produced by direct-current discharges (dc) [30, 31, 32, 33, 34], microwave discharges [35] and their afterglows [36], inductively coupled radio-frequency discharges [37], spark discharges [32], corona discharges [38, 39], dielectric barrier discharges [40] and ccrf discharges [41]. The infrared properties of dust particles synthesized within ccrf nitrogen / methane plasmas, at different mixing ratios, are studied for example in [42, 43].

Regardless of the specific application intended, a control upon the densities and/or energies of the main plasma species (via a modification in the operating conditions pressure and applied voltage or power) is essential to induce changes in the plasma reactivity, in view of either the reactor's optimization (in terms of processing rates and uniformity) or the tailoring of dust production. With this respect, the study of pure nitrogen discharges is a mandatory first step to understand the strong coupling between the discharge operating conditions and the plasma parameters, before moving to the (even more) complex kinetic schemes associated with $\mathrm{N}_{2}$ mixtures.

Although nitrogen plasmas have been studied for many years, and despite their growing interest for applications, there is only a partial knowledge about their behavior under ccrf discharge conditions. In fact, the majority of papers on $\mathrm{N}_{2}$ plasmas concern direct-current $[44,45,46,47,48,49,50]$, high-frequency (microwave) discharges [51, 
$52,53,54,55,56]$ and inductively coupled discharges [57], and focus essentially on their kinetic description. Usually, these are zero-dimensional self-consistent models, coupling the two-term electron Boltzmann equation (or just assuming a Maxwellian electron distribution function) to the rate balance equations of different vibrationally and electronically excited (molecular and atomic) states, yielding the electron energy distribution function (eedf), the vibrational distribution function (vdf) of the groundstate nitrogen molecules, and the populations of the excited species considered. These kinetic models play an important role in detailing the main mechanisms of (vibrational and electronic) excitation, dissociation, and ion production with $\mathrm{N}_{2}$ plasmas, but they offer only partial description of the charged and neutral species' dynamics, whose full analysis requires a multi-dimensional space-dependent approach. Other authors have adopted one-dimensional Monte-Carlo simulations to study the electron kinetics [58] and the coupling between the electron and the vibrational kinetics [59] of ccrf discharges in nitrogen. The approach was extended to a hybrid Monte-Carlo-fluid model [60], describing the electron kinetics and the chemistry of these discharge plasmas, and to Particle-in-Cell Monte-Carlo models calculating the eedf, to analyze its coupling with the vdf [61] or the electron heating mechanism within the space-charge sheaths [62].

In the particular case of non-equilibrium ccrf discharges in nitrogen, a self-consistent modelling strategy must account for the interplay between the transport of particles, in the presence of density gradients and the rf field, and their production / destruction due to kinetic mechanisms involving both electrons and heavy species. This modelling requires a two-dimensional (2D) description of the charged particle dynamics, to allow the calculation of the self-bias voltage (in the case of asymmetric discharges, with more surfaces grounded than driven) [63, 64], and to provide more reliable predictions for the profiles of the electron density and energy (hence, for the coupled electrical power). Also, in the case of molecular gases like nitrogen, the use of a comprehensive kinetic scheme is preferable: (i) to adequately describe electron-neutral collisions (especially with vibrational excited states), playing an essential role in coupling the plasma-gas system and establishing the final gas-phase chemical composition; and (ii) to deduce self-consistent electron parameters (transport and rate coefficients), whose calculation depends on the gas chemical composition via the eedf.

This paper studies ccrf discharges (at $f=13.56 \mathrm{MHz}$ frequency) in pure nitrogen, produced within cylindrical parallel-plate reactors (with radii $R=65$ and $69 \mathrm{~mm}$, and interelectrode distances $d=40$ and $33 \mathrm{~mm}$, for the experimental setups with the GREMI and the LATMOS laboratories, respectively, the latter corresponding to the PAMPRE experiment [29]), at $V_{\mathrm{rf}} \simeq 100-300 \mathrm{~V}$ applied voltages and $p \simeq 0.1-1$ mbar pressures. Under these conditions, the neutral gas is found close to room temperature $\left(T_{g} \simeq 350 \mathrm{~K}\right)$, as deduced from the rotational spectrum of the nitrogen second-positive system [41]. Figure 1 represents a schematic diagram of the plasma chamber and the external circuit with such reactors. In both reactors, the rf voltage is applied to the upper electrode through an L-type matching network and a blocking capacitor $C_{B} \simeq 760$ and $550 \mathrm{pF}$ for the GREMI and the LATMOS reactors, respectively (these values include the effects not 
only of the blocking capacitor but also of the stray capacitance and cables); a grounded counter electrode shields the back of the powered electrode and fixes a cylindrical lateral grid that confines the plasma. Mass flow controllers adjust the injection, through the driven electrode, of nitrogen and of argon traces added at constant $\mathrm{Ar} / \mathrm{N}_{2}$ flow ratio (of about $5 \%$ ), for the purpose of monitoring the evolution of electron-collision mechanisms. The nitrogen flow is kept equal to $25 \mathrm{sccm}$ in the GREMI reactor, while in the LATMOS reactor nitrogen is injected at flow rates between $4.6-98 \mathrm{sccm}$ to produce a pressure variation of $0.2-1.14$ mbar.

This work was driven by the need to provide answers to several challenging questions. (i) Is it possible to join a sophisticated 2D space-time dependent discharge code to a complex kinetic scheme to model ccrf discharges in pure nitrogen? (ii) Is it necessary to adopt such a complete description to provide an adequate modelling of these discharges? (iii) Is it reasonable to use such a highly-complex scheme, considering the uncertainties associated with some of the collisional data required? (iv) What are the model predictions for the dissociation degree of nitrogen and the evolution of the main radiative transitions with the excited $\mathrm{N}_{2}$ species? The strategy adopted to clarify these issues involves model validation by comparison between simulations and measurements performed on two similar (not twin) experimental setups and a sensitivity study of model results to variations of some key parameters.

Experimental diagnostics allow measuring the following quantities: (i) the applied rf voltage, using a high-voltage probe; (ii) the self-bias voltage; (iii) the effective power coupled to the plasma, taking into account circuit losses; (iv) the average electron density, using a resonant-cavity technique; (v) the intensities of radiative transitions with the nitrogen second-positive system (SPS) and first-negative system (FNS), and with the $811.5 \mathrm{~nm}$ atomic line of argon, using optical emission spectroscopy (OES) diagnostics. Modelling uses a hybrid code that couples a 2D time-dependent fluid module, describing the dynamics of charged particles (electrons and positive ions $\mathrm{N}_{2}^{+}$and $\mathrm{N}_{4}^{+}$), and a homogeneous (0D) kinetic module, describing the production and destruction of nitrogen (atomic and molecular) neutral species. The coupling between these modules adopts the local mean energy approximation $[65,66]$ to define space-time dependent electron parameters for the fluid module and to work-out space-time average rates for the kinetic module. Model results yield the self-consistent dc-bias voltage, the effective power coupled to the plasma, and the $2 \mathrm{D}$ spatial distributions for (i) the rf plasma potential, (ii) the densities and fluxes with the charged particles and the electron mean energy, and (iii) the densities of the most relevant nitrogen species.

\section{Experimental diagnostics}

The experimental analysis of the ccrf discharges in pure nitrogen resort to the following diagnostics: (i) electrical and plasma measurements of the applied rf voltage, the selfbias voltage, the power coupled to the plasma, and the average electron density (the latter is carried out on the GREMI reactor only); (ii) OES measurements of the nitrogen 
SPS and FNS band intensities, and of the $811.5 \mathrm{~nm}$ atomic-argon line intensity.

\subsection{Electrical and plasma measurements}

The applied rf voltage $V_{\mathrm{rf}}$, one of the main parameters defining the experimental work conditions, is measured onto the driven electrode by using a high-voltage probe connected to a digital oscilloscope. Once the plasma has reached its steady-state situation, the self-bias voltage $V_{\mathrm{dc}}$ is measured with a $10 \mathrm{M} \Omega$ voltmeter.

The power coupled to the plasma $W_{\text {eff }}$ is obtained adopting the so-called subtractive method [63, 67, 68, 69], which accounts for the power losses in the external circuitry by measuring the power at the output of the rf generator, for the same $V_{\mathrm{rf}}$, with and without plasma. Note that $W_{\text {eff }}$ is the quantity to consider in defining equivalent working conditions, for the comparison between simulations and measurements.

The diagnostic of the average electron density $\overline{n_{e}}$ uses the fact that the reactor chamber, with its lateral metal grid, acts as a resonant cavity in the microwave range. We prefer this space-average diagnostic to local probe measurements with compensation, since the latter are much more intrusive under ccrf conditions. By placing two axisymmetric electrically-insulated loop antenna at the bottom of the system [41], one can measure the shift in the resonance frequency of the cavity, with and without plasma, to deduce the on-axis electron density $n_{e_{0}}$ according to the relation $[70,71,72,73]$

$$
n_{e_{0}}=A 8 \pi^{2} f_{\text {res }}^{2} \frac{\Delta f_{\mathrm{res}}}{f_{\mathrm{res} 0}} \frac{m_{e} \varepsilon_{0}}{e^{2}} .
$$

In equation (1), $f_{\text {res }}$ and $f_{\text {res0 }}$ are the resonant frequency with and without plasma, respectively, $\Delta f \equiv\left|f_{\text {res }}-f_{\text {res0 }}\right|$ is the frequency shift, $e$ and $m_{e}$ are the electron charge and mass, respectively, $\varepsilon_{0}$ is the vacuum permittivity, and $A$ is a geometrical factor that depends on the electron density profile and on the electromagnetic mode adopted in measurements which, in our case, is found in the range $1-1.8$. For simplicity, we have taken $A=1$ (which corresponds to assume a constant electron density profile) since this approximation affects the value of $\overline{n_{e}} \simeq n_{e_{0}}$ by less than $10 \%$.

The value of $f_{\text {res } 0}$ has proven to be a critical parameter in providing a good estimation of the electron density. For example, the resonance frequency can change due to the small dilation of the metallic chamber affected by gas heating, and therefore it is measured here just after the plasma extinction. The choice of the electromagnetic mode is also of consequence. In a previous work [41] we have adopted the $\mathrm{TM}_{210}$ mode, whose resonance frequency is found near that of other transverse magnetic (TM) modes; here, we preferred the $\mathrm{TM}_{010}$ mode because it appears isolated and because its electric field distribution presents an on-axis maximum, coinciding with the position of the electron density maximum, hence allowing for measurements with higher sensitivity (in this case, reaching $\overline{n_{e}}$ values as low as $6 \times 10^{13} \mathrm{~m}^{-3}$ ). 


\subsection{Spectroscopic measurements}

OES diagnostics use an UV-VIS-NIR monochromator with a $60 \mathrm{~cm}$ focal length in both the LATMOS and the GREMI laboratories. These systems measure the intensities of the following radiative transitions: (i) the $370-438 \mathrm{~nm}$ bands with the SPS $\mathrm{N}_{2}\left(\mathrm{C}^{3} \Pi_{u}, \mathrm{v}\right) \rightarrow \mathrm{N}_{2}\left(\mathrm{~B}^{3} \Pi_{g}, \mathrm{v}^{\prime}\right)$ and the FNS $\mathrm{N}_{2}^{+}\left(\mathrm{B}^{2} \Sigma_{u}, \mathrm{v}^{\prime}\right) \rightarrow \mathrm{N}_{2}^{+}\left(\mathrm{X}^{2} \Sigma_{g}, \mathrm{w}^{\prime}\right)$ [74]. Of these,

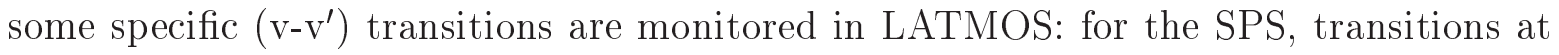
$\Delta \mathrm{v} \equiv \mathrm{v}^{\prime}-\mathrm{v}=2,3$, for $\mathrm{v}=0,1,2$ and at $\Delta \mathrm{v}=4$, for $\mathrm{v}=0,1,2,3,4$, of which the most intense one is the $380.5 \mathrm{~nm} \operatorname{SPS}(0-2)$; for the FNS, transitions (0-0), (0-1) and (1-2), of which the most intense one is the $391.4 \mathrm{~nm} \operatorname{FNS}(0-0)$; (ii) the $811.5 \mathrm{~nm} \operatorname{Ar}\left(4 \mathrm{p}[5 / 2]_{3}\right)$ $\rightarrow \operatorname{Ar}\left(4 \mathrm{~s}[3 / 2]_{2}\right)$ atomic line of argon, chosen because it presents no overlapping with nitrogen bands. As mentioned, argon is admitted at $\sim 5 \% \mathrm{Ar} / \mathrm{N}_{2}$ flow ratio in LATMOS or at percentages that ensure a constant 25 sccm nitrogen flow in GREMI. Argon acts as an actinomer: because its levels are populated by electron-collisions, the evolution of their line intensities is assumed to represent that of electron mechanisms.

\section{Model description}

To model ccrf discharges in pure nitrogen, we have developed a hybrid code coupling a 2D $(r, z)$ time-dependent fluid module (assuming azimuthal symmetry due to the configuration of the rf reactor) to a very complete 0D kinetic module. The fluid module solves the continuity and momentum-transfer equations for electrons and positive ions $\mathrm{N}_{2}^{+}(\mathrm{X}), \mathrm{N}_{2}^{+}(\mathrm{B})$ and $\mathrm{N}_{4}^{+}$, the electron mean energy transport equations (the ions are assumed to be thermalized at gas temperature), and Poisson's equation for the rf electric potential. The kinetic module solves the two-term homogeneous and stationary electron Boltzmann equation (accounting for inelastic collisions from ground-state molecules and atoms, and inelastic and superelastic collisions involving vibrationally excited states) and the rate balance equations of 45 vibrational excited states with the ground-state $\mathrm{N}_{2}\left(\mathrm{X}^{1} \Sigma_{g}^{+}, \mathrm{v}=0-45\right), 7$ electronic states $\mathrm{N}_{2}\left(\mathrm{~A}^{3} \Sigma_{u}^{+}, \mathrm{B}^{3} \Pi_{g}, \mathrm{C}^{3} \Pi_{u}, \mathrm{a}^{\prime 1} \Sigma_{u}^{-}, \mathrm{a}^{1} \Pi_{g}, \mathrm{w}^{1} \Delta_{u}\right.$, $\left.\mathrm{a}^{\prime \prime 1} \Sigma_{g}^{+}\right)$with the nitrogen molecule, and 3 electronic states $\mathrm{N}\left({ }^{4} \mathrm{~S},{ }^{2} \mathrm{D},{ }^{2} \mathrm{P}\right)$ with nitrogen atoms.

\subsection{The fluid module}

The fluid module is similar to that used to model ccrf discharges in hydrogen $[75,76]$. In particular, the flux equations (for both particle and energy) adopt the stationary driftdiffusion approximation, with the introduction of an effective field [77] in the specific case of the ions. Details about the validity of this approach are discussed in [65].

The continuity and the momentum-transfer equations for charged-particles $\alpha$ ( $\alpha=$ $e, i$ for electrons and ions, respectively) are given by

$$
\frac{\partial n_{\alpha}}{\partial t}=-\frac{1}{r} \frac{\partial\left(r \Gamma_{\alpha_{r}}\right)}{\partial r}-\frac{\partial \Gamma_{\alpha_{z}}}{\partial z}+S_{\alpha}
$$




$$
\Gamma_{\alpha_{q}}= \pm\left(\mu_{\alpha} N\right) n_{\alpha} \frac{E_{\alpha_{q}}^{\mathrm{eff}}}{N}-\frac{1}{N} \frac{\partial\left[\left(D_{\alpha} N\right) n_{\alpha}\right]}{\partial q} \quad( \pm \text { for } e, i)
$$

Here, $n_{\alpha}(r, z, t)$ is the particle density; $\Gamma_{\alpha_{q}}(r, z, t)$ are the $q=r, z$ components of the particle flux; $S_{\alpha}(r, z, t)$ is the net production rate of particles due to kinetic mechanisms; $N=p / k_{B} T_{g}$ is the gas density ( $k_{B}$ is the Boltzmann's constant); $\left(\mu_{\alpha} N\right)(r, z, t)$ and $\left(D_{\alpha} N\right)(r, z, t)$ are the reduced mobility and diffusion coefficient, respectively; and $\left(E_{\alpha_{q}}^{\text {eff }} / N\right)(r, z, t)$ is the $q$ component of the reduced effective electric field. For the electrons $E_{e_{q}}^{\text {eff }}=E_{q}$, corresponding to the rf electric field, whereas for the ions $E_{i_{q}}^{\text {eff }}$ is calculated using [65]

$\frac{\partial\left(E_{i_{q}}^{\mathrm{eff}} / N\right)}{\partial t}=-\frac{1}{\mu_{i} N}\left(v_{i_{r}} \frac{\partial}{\partial r}+v_{i_{z}} \frac{\partial}{\partial z}\right) v_{i_{q}}-\frac{\nu_{i}}{\mu_{i} N} v_{i_{q}}+\nu_{i}^{c}\left(\frac{E_{q}}{N}-\frac{E_{i_{q}}^{\mathrm{eff}}}{N}\right)$,

where $v_{i_{q}} \equiv \Gamma_{i_{q}} / n_{i}$ is the $q$ component of the $i$-ion drift velocity, $\nu_{i} \equiv S_{i} / n_{i}$ is the $i$-ion net production frequency, and $\nu_{i}^{c}$ is the ion-neutral momentum-transfer collision frequency. The latter relates to the ion mobility, according to $\mu_{i} N=e /\left[m_{i}\left(\nu_{i}^{c} / N\right)\right]$ with $m_{i}$ the $i$-ion mass.

The electron mean energy transport equations are given by

$$
\begin{aligned}
\frac{\partial\left(n_{e} \varepsilon\right)}{\partial t} & =-\frac{1}{r} \frac{\partial\left(r \Gamma_{\varepsilon_{r}}\right)}{\partial r}-\frac{\partial \Gamma_{\varepsilon_{z}}}{\partial z}-\vec{\Gamma}_{e} \cdot \vec{E}-S_{\varepsilon} \\
\Gamma_{\varepsilon_{q}} & =-\left(\mu_{\varepsilon} N\right) \varepsilon n_{e} \frac{E_{q}}{N}-\frac{1}{N} \frac{\partial\left[\left(D_{\varepsilon} N\right) \varepsilon n_{e}\right]}{\partial q},
\end{aligned}
$$

where $\varepsilon(r, z, t)$ is the electron mean energy, $\Gamma_{\varepsilon_{q}}(r, z, t)$ is the $q$ component of the electron energy flux, $\left(\mu_{\varepsilon} N\right)(r, z, t)$ and $\left(D_{\varepsilon} N\right)(r, z, t)$ are the reduced mobility and diffusion coefficient for energy transport, respectively, and $S_{\varepsilon}(r, z, t)$ is the net power density lost by the electrons due to (elastic ands inelastic) collisions. Equation (4a) describes the rate of change of the electron energy density $n_{e} \varepsilon$ as the result of (by order of the terms on the right-and side of the equation) convection [corresponding to the transport of energy due to the drift-diffusion electron motion, see equation (4b)] conduction (corresponding to the transfer of energy from the rf electric field to the electrons) and friction (corresponding to the electron energy dissipation in collisions).

The fluid module is closed by Poisson's equation for the rf potential $V(r, z, t)$

$$
\frac{1}{r} \frac{\partial}{\partial r}\left(r \frac{\partial V}{\partial r}\right)+\frac{\partial^{2} V}{\partial z^{2}}=-\frac{e}{\varepsilon_{0}}\left(\sum_{i} n_{i}-n_{e}\right) \text {. }
$$

Equation (5) relates the space-time separation of charged particles to the rf electric field $\vec{E}(r, z, t)=-\vec{\nabla}_{r, z} V(r, z, t)$.

Equations (2a)-(5) are solved within the plasma reactor (corresponding to a $2 \mathrm{D}$ workspace delimited by the discharge axis at $r=0$, the grounded lateral grid at $r=R$, the driven electrode at $z=0$, and the grounded electrode at $z=d$, see Fig. 1), subject to the following boundary conditions. At the reactor axis symmetry boundary conditions are given by

$$
\frac{\partial n_{\alpha}}{\partial r} \quad=0 \quad(\alpha=e, i)
$$




$$
\begin{aligned}
& \frac{\partial\left(n_{e} \varepsilon\right)}{\partial r}=0 \\
& \frac{\partial V}{\partial r}=0 .
\end{aligned}
$$

Flux conditions for totally absorbing boundaries are imposed at the different electrodes and walls using $[65,66]$

$$
\begin{aligned}
& \left(\Gamma_{e}\right)_{\perp}=\frac{1}{2} n_{e}\langle v\rangle-\xi_{e} \sum_{i}\left(\Gamma_{i}\right)_{\perp} \\
& \left(\Gamma_{\varepsilon}\right)_{\perp}=\frac{1}{2} n_{e}\langle v u\rangle-\xi_{e} \varepsilon \sum_{i}\left(\Gamma_{i}\right)_{\perp} \\
& \left(\Gamma_{i}\right)_{\perp}=\frac{1}{4} n_{i} v_{\mathrm{th}_{i}}+\beta_{i} n_{i}\left(\mu_{i} N\right) \frac{E_{i_{\perp}}^{\mathrm{eff}}}{N} .
\end{aligned}
$$

In these equations, $\perp$ relates to the flux component perpendicular to the boundary; $\langle v\rangle$ and $\langle v u\rangle$ represent the average values of $v$ and $v u$ over the eedf, respectively; $\xi_{e}$ is the secondary electron emission coefficient (here, we assume that secondary emission occurs at the driven electrode only, with $\left.\xi_{e}=0.1-1\right) ; v_{\mathrm{th}_{i}}=\left(8 k_{B} T_{g} / \pi m_{i}\right)^{1 / 2}$ is the thermal velocity for the $i$-ion species, and

$$
\beta_{i}= \begin{cases}1 & \text { if } E_{i_{\perp}}^{\mathrm{eff}} \text { is directed towards the wall } \\ 0 & \text { if } E_{i_{\perp}}^{\mathrm{eff}} \text { is in the opposite direction to the wall } .\end{cases}
$$

Note that conditions $(7 a)-(7 b)$, for the electron particle and energy, are obtained using the expansion that writes the electron distribution function as a combination of two components (isotropic and anisotropic) [66], whereas condition (7c) for the ions separates between their thermal (isotropic) and drift (anisotropic) motion. Finally, the potential at each physical boundary satisfies

$$
V= \begin{cases}V_{\mathrm{dc}}+V_{\mathrm{rf}} \cos (\omega t) & \text { at driven electrode } \\ 0 & \text { at grounded electrode and walls }\end{cases}
$$

where $\omega=2 \pi f, V_{\mathrm{rf}}$ is the peak voltage applied to the driven electrode, and $V_{\mathrm{dc}}$ is an offset potential termed self-bias voltage, that develops in the case of an asymmetric reactor (with more surfaces grounded than driven). The value of the self-bias voltage is updated, after each RF period $T_{\text {rf }}$, using

$$
V_{\mathrm{dc}}=V_{\mathrm{dc}}(t)+\int_{t}^{t+T_{\mathrm{rf}}} \frac{1}{C_{B}} I_{\mathrm{c}}\left(t^{\prime}\right) d t^{\prime}
$$

where $I_{c}(t)=C_{B}\left(d V_{C}(t) / d t\right)$ is the current across the blocking capacitor $C_{B}$ (see Fig. 1 ). This current can be evaluated by integrating the axial component of the conduction current density over the surface of the driven electrode

$$
I_{\mathrm{c}}(t)=\int_{0}^{R} J_{\mathrm{C}_{z}}(r, 0, t) 2 \pi r d r
$$

introducing

$$
\overrightarrow{J_{\mathrm{c}}}=e\left(\sum_{i} \Gamma_{i}-\Gamma_{e}\right) \simeq \sigma \vec{E}+\vec{J}_{\mathrm{diff}}
$$


where $\sigma \equiv e\left(\mu_{e} n_{e}+\sum_{i} \mu_{i} n_{i}\right)$ is the total plasma conductivity (defined by assuming $\vec{E}_{i}^{\text {eff }} \simeq \vec{E}$ ) and $\vec{J}_{\text {diff }}$ is the net diffusion current defined according to [cf. equation $(2 b)$ ]

$$
\vec{J}_{\text {diff }} \equiv-e \sum_{i}\left(D_{i} N\right) \frac{\vec{\nabla}}{N} n_{i}+e \frac{\vec{\nabla}}{N}\left[\left(D_{e} N\right) n_{e}\right] .
$$

The total current $I_{\mathrm{t}}$ is obtained by adding the contributions of the displacement current $I_{\mathrm{D}}$ and the conduction current $I_{\mathrm{c}}$

$I_{\mathrm{t}}(t)=I_{\mathrm{c}}(t)+I_{\mathrm{D}}(t)=\int_{0}^{R} J_{\mathrm{c}_{z}}(r, 0, t) 2 \pi r d r+\int_{0}^{R} \varepsilon_{0}\left(\frac{\partial E_{z}}{\partial t}\right)(r, 0, t) 2 \pi r d r \quad$,

and it is used to evaluate the effective electrical power coupled to the plasma

$$
W_{\text {eff }} \equiv \frac{1}{T_{\mathrm{rf}}} \int_{0}^{T_{\mathrm{rf}}}\left[V_{\mathrm{dc}}+V_{\mathrm{rf}} \cos (\omega t)\right] I_{\mathrm{t}}(t) d t
$$

The electron parameters (transport and rate coefficients) in equations $(2 a)-(4 b)$ are calculated as integrals of cross sections over the eedf (see reference [66] for more details on these expressions). As mentioned, the space-time dependence of these parameters is defined by adopting the so-called local mean energy approximation [65, 66], which assumes that the space-time variation of the eedf, $f(u, r, z, t)$, is introduced via the local ionization degree and the electron mean energy profile, i.e. $(u$ is the electron kinetic energy)

$$
f(u, r, z, t)=f(u)_{\mid \varepsilon(r, z, t), n_{e}(r, z, t)} .
$$

The procedure is the following: first, the homogeneous and stationary eedf, $f(u)$, is calculated from the kinetic module (see section 3.2) for various values of $n_{e} / N$ (as input) and of $\varepsilon_{\text {eedf }}=\int_{0}^{\infty} u^{3 / 2} f(u) d u$ (as output); second, the eedfs obtained are used to calculate the different electron parameters, which are then tabulated as a function of $n_{e} / N$ and $\varepsilon_{\text {eedf }}$; third, the local values of the electron density and mean energy, $n_{e}(r, z, t)$ and $\varepsilon(r, z, t)$, obtained from the solution to the electron particle and energy balance equations $(2 a)$ and $(4 a)$, are used in the table constructed to define the space-time map of the electron transport and rate coefficients.

The ion transport parameters in equations $(2 a)-(2 b)$ are as follows. For the ion mobilities, a dependence on the reduced effective electric field was introduced, according to $[78,79]$ (values in $\mathrm{V}^{-1} \mathrm{~cm}^{2} \mathrm{~s}^{-1}$, referred to a standard gas density of $2.69 \times 10^{19} \mathrm{~cm}^{-3}$; $\left.1 \mathrm{Td}=10^{-17} \mathrm{~V} \mathrm{~cm}^{2}\right)$

$$
\mu_{N_{2}^{+}(X, B)}=\left\{\begin{aligned}
1.90 & \text { if } E_{N_{2}^{+}}^{\mathrm{eff}} \leq 7 \mathrm{Td} \\
0.29+0.71 \exp \left[-\frac{E_{N_{2}^{+}}^{\mathrm{eff}}}{2531.01}\right] & \\
+0.93 \exp \left[-\frac{E_{N_{2}^{+}}^{\mathrm{eff}}}{257.37}\right] & \text { if } E_{N_{2}^{+}}^{\mathrm{eff}} \geq 7 \mathrm{Td}
\end{aligned}\right.
$$




$$
\mu_{N_{4}^{+}}=\left\{\begin{array}{cl}
2.31 & \text { if } E_{N_{4}^{+}}^{\mathrm{eff}} \leq 7 \mathrm{Td} \\
2.25+0.55 \exp \left[\frac{E_{N_{4}^{+}}^{\mathrm{eff}}}{30.93}\right]-0.10 \exp \left[-\frac{E_{N_{4}^{+}}^{\mathrm{eff}}}{3.44}\right] & \\
-0.49 \exp \left[\frac{E_{N_{4}^{+}}^{\mathrm{eff}}}{30.06}\right] & \text { if } 7 \mathrm{Td} \leq E_{N_{4}^{+}}^{\mathrm{eff}} \leq 100 \mathrm{Td} \\
0.47+1.15 \exp \left[-\frac{E_{N_{4}^{+}}^{\mathrm{eff}}}{2531.01}\right] & \\
+1.50 \exp \left[-\frac{E_{N_{4}^{+}}^{\mathrm{eff}}}{257.37}\right] & \text { if } E_{N_{4}^{+}}^{\mathrm{eff}} \geq 100 \mathrm{Td} .
\end{array}\right.
$$

For the ion diffusion coefficients we have used the values proposed in [80] $D N_{N_{2}^{+}(X, B)}=$ $1.7 \times 10^{18}\left(T_{g} / 273\right)^{1 / 2}$ and $D N_{N_{4}^{+}}=1.5 \times 10^{18}\left(T_{g} / 273\right)^{1 / 2} \mathrm{~cm}^{-1} \mathrm{~s}^{-1}$.

\subsection{The kinetic module}

The kinetic module solves the space-time average rate balance equations, for the different neutral (molecular and atomic) species considered.

Generally, the rate balance equation for species $k$ is given by (for the axis-symmetric geometry considered here)

$$
\frac{\partial n_{k}}{\partial t}+\frac{1}{r} \frac{\partial\left(r \Gamma_{k_{r}}\right)}{\partial r}+\frac{\partial \Gamma_{k_{z}}}{\partial z}=S_{k}
$$

where $n_{k}$ and $\Gamma_{k_{q}}$ are the species density and flux $q$-component, respectively, and $S_{k}$ is a source term accounting for the net production of $k$-species

$$
\begin{aligned}
S_{k} & =\sum_{\alpha, l} C_{\alpha l, k} n_{\alpha} n_{l}-n_{k} \sum_{\alpha} C_{\alpha k}^{\mathrm{tot}} n_{\alpha}+\sum_{l, m} K_{l m, k} n_{l} n_{m}-n_{k} \sum_{l} K_{k l}^{\mathrm{tot}} n_{l} \\
& +\sum_{l} A_{l, k} n_{l}-n_{k} A_{k}^{\mathrm{tot}} .
\end{aligned}
$$

In equation (17), $C_{\alpha l, k}$ and $K_{m l, k}$ are the rate coefficients for the production of species $k$ from collisions of neutral species $l$ with charged species $\alpha$ and neutral species $m$, respectively; $C_{k \alpha}^{\text {tot }}$ and $K_{k l}^{\text {tot }}$ are the rate coefficients for the total destruction of species $k$ from collisions with charged species $\alpha$ and neutral species $l$, respectively; $A_{l, k}$ is the production frequency of $k$-species from the radiative decay of $l$-species; and $A_{k}^{\text {tot }}$ is the total destruction frequency of $k$-species by radiative decay.

In order to limit the calculation runtime (due to the high number of species and kinetic processes considered), the kinetic module solves the stationary space-average version of equations (16)

$$
\begin{aligned}
& \frac{1}{\left(R^{2} / 2\right) d}\left\{\int_{0}^{d} R \Gamma_{r_{k}}(R, z) d z+\int_{0}^{R}\left[\Gamma_{z_{k}}(r, d)-\Gamma_{z_{k}}(r, 0)\right] r d r\right\} \\
& =\frac{1}{\left(R^{2} / 2\right) d} \int_{0}^{R} \int_{0}^{d} S_{k} d z r d r .
\end{aligned}
$$


The use of a stationary approach to describe the neutral (heavy) species dynamics is justified, considering the long relaxation times associated with both their transport $\left[n_{k} /\left(D_{k} \nabla^{2} n_{k}\right) \sim \delta^{2} / D_{k} \sim 0.4 \mathrm{~ms}\right.$, for a diffusion coefficient $D_{k} \sim 10^{3} \mathrm{~cm}^{2} \mathrm{~s}^{-1}$ and a thickness of the space-charge sheath $\delta \sim 1 \mathrm{~cm}]$ and their kinetic mechanisms $\left[1 /\left(n_{e} C_{e k}\right) \sim 0.1-1 \mathrm{~s}\right.$, for an electron density $n_{e} \sim 10^{9} \mathrm{~cm}^{-3}$ and excitation rate coefficients $C_{e k} \sim 10^{-8}-10^{-10} \mathrm{~cm}^{3} \mathrm{~s}^{-1}$, when compared with the rf period of $1 / f \sim 74$ ns.

The boundary fluxes in equation (18) are set to satisfy the Milne's condition [81, 82]

$$
\left.\Gamma_{q_{k}}\right|_{\mathrm{wall}}=-\left.D_{k} \frac{\partial n_{k}}{\partial q}\right|_{\mathrm{wall}}=\left.\gamma_{k}^{\prime} \frac{v_{\mathrm{th}_{k}}}{4} n_{k}\right|_{\mathrm{wall}} \quad(q=r, z)
$$

where $\gamma_{k}^{\prime} \equiv \gamma_{k} /\left(1-\gamma_{k} / 2\right)$ with $\gamma_{k}$ the $k$-species' wall loss probability.

By using equations (17) and (19) into (18) one obtains

$$
\begin{aligned}
& \frac{1}{\left(R^{2} / 2\right) d} \frac{\gamma_{k}}{1-\gamma_{k} / 2} \frac{v_{\mathrm{th}_{k}}}{4}\left\{\int_{0}^{d} R n_{k}(R, z) d z+\int_{0}^{R}\left[n_{k}(r, d)-n_{k}(r, 0)\right] r d r\right\} \\
& \simeq \sum_{\alpha}\left[\sum_{l} \overline{C_{\alpha l, k} n_{\alpha}} \overline{n_{l}}-\overline{C_{\alpha k}^{\mathrm{tot} n_{\alpha}}} \overline{n_{k}}\right]+\sum_{l}\left[\sum_{m} K_{l m, k} \overline{n_{l} n_{m}}-K_{k l}^{\mathrm{tot}} \overline{n_{k} n_{l}}\right] \\
& +\sum_{l} A_{l, k} \overline{n_{l}}-A_{k}^{\mathrm{tot}} \overline{n_{k}}
\end{aligned}
$$

where the average quantities $\bar{X}\left(X=n_{k}, n_{k} n_{l}, C_{\alpha l, k} n_{\alpha}, \ldots\right)$ are defined as

$$
\bar{X} \equiv \frac{\int_{0}^{d} \int_{0}^{R} X r d r d z}{\left(R^{2} / 2\right) d}
$$

The space-averages in equation (20) are obtained as follows. The averages of the charged-particle collision frequencies $\left(\overline{C_{\alpha l, k} n_{\alpha}}\right.$ and $\left.\overline{C_{\alpha k}^{\text {tot }} n_{\alpha}}\right)$ are calculated using the space-dependent distributions obtained from the solution to the fluid module; the averages of the heavy species densities $\left[\overline{n_{k}}, \overline{n_{k} n_{l}}\right.$, and the integrals on the left-hand side of equation (18)] are calculated imposing the following density profiles: (i) a constant profile for ground-state species $\mathrm{N}_{2}\left(\mathrm{X}^{1} \Sigma_{g}^{+}, \mathrm{v}\right)$ and $\mathrm{N}\left({ }^{4} \mathrm{~S}\right)$; (ii) a two-region profile (see Fig. 2) that distinguishes between the plasma bulk (with a spatially homogeneous density) and the plasma sheaths (where the density decreases linearly), for species $\mathrm{N}_{2}\left(\mathrm{~A}^{3} \Sigma_{u}^{+}, \mathrm{a}^{1} \Sigma_{u}^{-}, \mathrm{a}^{1} \Pi_{g}, \mathrm{w}^{1} \Delta_{u}\right)$ and $\mathrm{N}\left({ }^{2} \mathrm{D},{ }^{2} \mathrm{P}\right)$, that are transported by diffusion and are totally recombined at the walls (i.e., for which $\gamma_{k}^{\prime}=1$ ). The $2 \mathrm{D}$ version of this profile is given by

$$
\begin{aligned}
& n_{k}(r, z)=n_{b_{k}} h_{k}(r) g_{k}(z) \\
& h_{k}(r)= \begin{cases}1 & , 0 \leq r<R-\delta_{R} \\
1-\frac{1-f_{r_{k}}}{\delta_{R}}\left(r-R+\delta_{R}\right) & , R-\delta_{R} \leq r \leq R\end{cases} \\
& g_{k}(z)= \begin{cases}f_{0_{k}}+\frac{1-f_{0_{k}}}{\delta_{0}} z & , 0 \leq z \leq \delta_{0} \\
1 & , \delta_{0}<z<d-\delta_{d} \\
1-\frac{1-f_{d_{k}}}{\delta_{d}}\left(z-d+\delta_{d}\right) & , d-\delta_{d} \leq z \leq d\end{cases}
\end{aligned}
$$


where $n_{b_{k}}$ is the bulk density; $\delta_{R}, \delta_{0}$, and $\delta_{d}$ are the sheath thickness near the grid, the driven electrode, and the grounded electrode, respectively; and the functions $f_{r_{k}}, f_{0_{k}}$, and $f_{d_{k}}$ correspond to the ratio of the wall density to the bulk density, calculated at $r=R, z=0$, and $z=d$ using boundary condition (19), i.e.

$$
f_{x_{k}} \equiv \frac{\left.n_{k}\right|_{\mathrm{wall}}}{n_{b_{k}}}=\frac{\frac{4}{\gamma_{k}^{\prime} v_{\mathrm{th}_{k}}}}{\frac{4}{\gamma_{k}^{\prime} v_{\mathrm{th}_{k}}}+\frac{\delta_{x}}{D_{k}}} \quad(x=r, 0, d) .
$$

As we have associated the boundary layers for neutral particles with the discharge space-charge sheaths, the quantities $\delta_{R}, \delta_{0}$, and $\delta_{d}$ are estimated in the fluid module as the time-average thicknesses of the regions where the rf field exhibits strong relative gradients. In particular, the sheath edges are taken at positions where the corresponding (axial or radial) reduced electric fields are equal to $10 \%$ of the wall field $\left(E_{z} / N\right)(0,0)$, $\left(E_{z} / N\right)(0, d)$ or $\left(E_{r} / N\right)(R, d / 2)$. Note that equations $(22 a)-(23)$ allow calculating the space-average integrals in equation (20) also for the heavy species with constant density profiles, by setting $\delta_{x}=0$.

As mentioned, the kinetic module solves the rate balance equations of 45 vibrational states (with the ground-state molecule) and 10 electronic states ( 7 for molecules and 3 for atoms), coupled to the two-term homogeneous and stationary electron Boltzmann equation, accounting for inelastic collisions from ground-state molecules and atoms, and inelastic and superelastic collisions involving vibrationally excited states (only the first 10 states are considered, see Table 1). This module adopts the kinetic scheme proposed in reference [80], where details about the choices of the different mechanisms and rates can be found. Tables 1-2 summarize the kinetic reactions for the molecular species and the atomic species, respectively, used in writing the source terms (17) and in solving the electron Boltzmann equation. In these tables, the double arrow $\longleftrightarrow$ indicates that second-kind collisions are also considered for the reactions where it appears. The cross sections for the various electron-neutral collisional processes (appearing in the electron Boltzmann equation) are taken from [83, 46, 47] and references therein. The cross sections for superelastic collisions are obtained from those for the corresponding inelastic processes by using Klein-Rosseland's formula [84].

The vibrational states of ground-state $\mathrm{N}_{2}\left(\mathrm{X}^{1} \Sigma_{g}^{+}, \mathrm{v}\right)$ molecules play a central role in nitrogen discharges. The kinetics of vibrational states includes excitation/deexcitation mechanisms due to electron impact collisions (e-V) transitions and heavy species collisions, involving an energy transfer via vibrational-translational (V-T, with both molecules and atoms) and vibrational-vibrational (V-V) interactions. Moreover, several vibrational states are involved in heavy-particle reactions, some of them leading to dissociation (see Tables 1-2). This work adopts the SSH (Schwartz, Slawsky and Herzfeld) theory $[84,85,86,87,88]$ to write the rate coefficients for (i) the V-T mechanisms with molecules

$$
P_{\mathrm{v}, \mathrm{v}-1}=\mathrm{v}\left(\frac{1-\chi_{e}^{\mathrm{N}_{2}}}{1-\chi_{e}^{\mathrm{N}_{2} \mathrm{v}}}\right) \frac{F\left(Y_{\mathrm{v}, \mathrm{v}-1}\right)}{F\left(Y_{1,0}\right)} P_{1,0}
$$




$$
\begin{aligned}
& P_{\mathrm{v}-1, \mathrm{v}}=P_{\mathrm{v}, \mathrm{v}-1} \exp \left[-\frac{\Delta E_{\mathrm{v}, \mathrm{v}-1}}{k_{B} T_{g}}\right] \\
& Y_{\mathrm{v}, \mathrm{v}-1}=\frac{\pi L}{\hbar}\left(\frac{M}{4 k_{B} T_{g}}\right)^{1 / 2} \Delta E_{\mathrm{v}, \mathrm{v}-1}
\end{aligned}
$$

with the normalization [89]

$$
P_{1,0}\left(\mathrm{~cm}^{3} \mathrm{~s}^{-1}\right)=\frac{1.07 \times 10^{-12} T_{g}^{3 / 2}}{0.2772 T_{g}-80.32+35.5\left(\frac{\mathrm{v}-1}{39}\right)^{0.8}} F\left(Y_{1,0}\right),
$$

and (ii) the V-V mechanisms

$$
\begin{aligned}
& Q_{\mathrm{v}, \mathrm{v}-1}^{\mathrm{w}-1, \mathrm{w}}=\mathrm{vw}\left(\frac{1-\chi_{e}^{\mathrm{N}_{2}}}{1-\chi_{e}^{\mathrm{N}_{2} \mathrm{v}}}\right)\left(\frac{1-\chi_{e}^{\mathrm{N}_{2}}}{1-\chi_{e}^{\mathrm{N}_{2} \mathrm{w}}}\right) \frac{F\left(Y_{\mathrm{v}, \mathrm{v}-1}^{\mathrm{w}-1, \mathrm{w}}\right)}{F\left(Y_{1,0}^{0,1}\right)} Q_{1,0}^{0,1} \\
& Q_{\mathrm{v}-1, \mathrm{v}}^{\mathrm{w}, \mathrm{w}-1}=Q_{\mathrm{v}, \mathrm{v}-1}^{\mathrm{w}-1 \mathrm{w}} \exp \left[-\frac{\Delta E_{\mathrm{v}, \mathrm{v}-1}^{\mathrm{w}-1, \mathrm{w}}}{k_{B} T_{g}}\right] \\
& Y_{\mathrm{v}, \mathrm{v}-1}^{\mathrm{w}-1, \mathrm{w}}=\frac{\pi L}{\hbar}\left(\frac{M}{4 k_{B} T_{g}}\right)^{1 / 2} \Delta E_{\mathrm{v}, \mathrm{v}-1}^{\mathrm{w}-1, \mathrm{w}}
\end{aligned}
$$

with the normalization [89]

$$
\begin{aligned}
& Q_{1,0}^{0,1}\left(\mathrm{~cm}^{3} \mathrm{~s}^{-1}\right)=\frac{6.35 \times 10^{-17} T_{g}^{3 / 2}}{\zeta} \\
& \zeta=\left\{\begin{array}{ll}
39.065-1.5625 \max [\mathrm{v}, \mathrm{w}] & , \max [\mathrm{v}, \mathrm{w}]<10 \\
25.2+24.1\left(\frac{\max [\mathrm{v}, \mathrm{w}]-10}{30}\right)^{3} & , \max [\mathrm{v}, \mathrm{w}] \geq 10
\end{array} .\right.
\end{aligned}
$$

The rate coefficients for the $\mathrm{V}-\mathrm{T}$ mechanisms with atoms consider the calculations of references $[90,91,92,93]$ to deduce the approximated formulae

$$
\begin{aligned}
& P_{\mathrm{v}, \mathrm{w}<\mathrm{v}}^{\mathrm{N}_{2}-\mathrm{v}}= \begin{cases}A_{0} \exp \left(-\frac{A_{1}}{\mathrm{v}}+\frac{A_{2}}{\mathrm{v}^{2}}\right) & , \mathrm{v}-\mathrm{w} \leq 5 \text { and } \mathrm{v} \geq \mathrm{v}^{\star} \\
0 & \text { otherwise }\end{cases} \\
& P_{\mathrm{w}<\mathrm{v}, \mathrm{v}}^{\mathrm{N}_{2}-\mathrm{N}}=P_{\mathrm{v}, \mathrm{w}<\mathrm{v}}^{\mathrm{N}_{2}-\mathrm{N}} \exp \left[-\frac{\Delta E_{\mathrm{v}, \mathrm{w}<\mathrm{v}}}{k_{B} T_{g}}\right]
\end{aligned}
$$

where the quantities $A_{0}, A_{1}, A_{2}$ and $\mathrm{v}^{\star}$ are given in Table 3 , for both reactive (with an atomic exchange between the two collision partners) and non-reactive (direct) collisions. In equations $(24 a)-(26 b)$

$$
F(Y)=\left\{\begin{array}{ll}
\frac{1}{2}\left[3-\exp \left(-\frac{2 Y}{3}\right)\right] \exp \left(-\frac{2 Y}{3}\right) & , 0 \leq Y \leq 20 \\
8\left(\frac{\pi}{3}\right)^{1 / 2} Y^{7 / 3} \exp \left(-3 Y^{2 / 3}\right) & , Y>20
\end{array} ;\right.
$$

$\Delta E_{\mathrm{v}, \mathrm{v}-1}=\hbar \omega^{\mathrm{N}_{2}}\left(1-2 \chi_{e}^{\mathrm{N}_{2}} \mathrm{v}\right)$ is the energy difference between two consecutive levels $\mathrm{v}$ and $\mathrm{v}-1 ; \Delta E_{\mathrm{v}, \mathrm{v}-1}^{\mathrm{w}-1, \mathrm{w}}=\hbar \omega^{\mathrm{N}_{2}} 2 \chi_{e}^{\mathrm{N}_{2}}|\mathrm{w}-\mathrm{v}|$ is the energy variation with the transition $\mathrm{v}$ to $\mathrm{v}-1$ and $\mathrm{w}-1$ to $\mathrm{w} ; \Delta E_{\mathrm{v}, \mathrm{w}<\mathrm{v}}$ is the energy difference between levels $\mathrm{v}$ and $\mathrm{w}<\mathrm{v}$; $\omega^{\mathrm{N}_{2}}=4.443 \times 10^{14} \mathrm{~s}^{-1}$ and $\chi_{e}^{\mathrm{N}_{2}}=6.073 \times 10^{-3}$ are parameters characterizing the anharmonic Morse's oscillator; $L=2 \times 10^{-11} \mathrm{~m}$ is the range of intermolecular forces; $M$ is the mass of the $\mathrm{N}_{2}$ molecule; and $\hbar \equiv h /(2 \pi)$ with $h$ the Planck's constant. Note that the rate coefficients $(24 b),(25 b)$ and $(26 b)$ for the inverse V-T (with molecules and atoms) 
and V-V reactions were obtained from $(24 a)$, (25a) and (26a) by detailed balancing. Note also that only single-quantum transitions (the most likely ones) are considered for $\mathrm{V}-\mathrm{V}$ and $\mathrm{V}-\mathrm{T}$ reactions with molecules, whereas multi-quantum transitions are accounted for in V-T exchanges with atoms. Vibrational dissociation by $\mathrm{V}-\mathrm{V}$ and $\mathrm{V}-\mathrm{T}$ processes is also included, as a transition from $\mathrm{v}=45$ to a pseudo-level in the continuum [94].

The kinetic scheme presented in Tables 1-2 depends on quantities whose knowledge is somewhat limited, such as the secondary electron emission coefficient $\xi_{e}$, the wall loss probability $\gamma_{k}^{\prime}$ of ground-state species $\mathrm{N}_{2}\left(\mathrm{X}^{1} \Sigma_{g}^{+}, \mathrm{v}\right)$ and $\mathrm{N}\left({ }^{4} \mathrm{~S}\right)$ (which may depend on the working conditions and / or the specific surface properties), and the branching ratios $b_{\text {ion }}$ for the associative ionization reactions (23)-(24) and $b_{\text {atom }}$ for the atomic walldeexcitations (47)-(48). There is a difference in the nature of the walls, between the GREMI and the LATMOS reactors: both are essentially made of stainless-steel, but the counter-electrode with the LATMOS reactor is in aluminum alloy. With no specific data for $\gamma_{k}^{\prime}$, accounting for the interaction of species with different reactor walls, the quality of comparison between model results and measurements is probably affected. For the simulations in section 4 we have taken $\xi_{e}=0, \gamma_{\mathrm{N}_{2}}=4.5 \times 10^{-4}, \gamma_{\mathrm{N}}=10^{-3}$, and $b_{\text {ion }}=b_{\text {atom }}=0.5$. The sensitivity of the model results to these input parameters will be discussed in section 4 .

\subsection{Model solution}

The solution to the model iterates between the kinetic and the fluid modules. Figure 3 presents the flowchart of the numerical workflow used in the simulations.

Typically the kinetic module runs every ten rf periods, knowing the space-timeaverage values of the charged particle densities and of the electron rate-coefficients / rates for the production / destruction of each neutral species. Reducing, by a factor of five, the frequency adopted for updating the kinetic module speeds up calculations by a factor of two, with negligible changes (below 2\%) in the main quantities calculated. Results are also not affected if the kinetic module is updated more frequently instead, but the calculation time is heavily degraded in this case. The stationary solution to the set of (non-linear) rate balance equations (20) is obtained combining a semi-implicit timerelaxation technique with an iterative matrix-inversion method. Convergence ensures relative variations of less than $10^{-6}$ for the densities of all neutral species and is achieved after several hundred iterations. The new chemical composition of the gas phase is then used as input data to the homogeneous electron Boltzmann equation, allowing updating the set of electron parameters (transport and rate coefficients). The spacetime dependence of the electron parameters is defined by the local electron mean energy approximation (see section 3.1), and the spatial variation of the neutral species densities is tailored using the profiles given by equations $(22 a)-(22 c)$; then, these quantities are used in the solution to the fluid module. Replacing (22a)-(22c) with constant profiles for the populations of the molecular and the atomic excited species yields an increase of 
$5 \%, 8 \%$ and $2.5 \%$ for the charged particle densities, the self-bias voltage and the coupled power, respectively, and a $2 \%$ variation in the density of neutral species.

The charged particle transport equations (2a)-(4b) and Poisson's equation (5) are discretized using a second order finite difference representation that includes boundary conditions (6a)-(8). In order to correctly describe the space-charge sheaths, near the integration boundaries, the flux equations $(2 b),(4 b)$ are discretized using the ScharfetterGummel exponential scheme [95]. In this work, Poisson's equation is numerically solved by direct matrix methods, and the charged particle transport equations are integrated by adopting a semi-implicit splitting method, that uses a Crank-Nicholson algorithm with an integration time step controlled by the Courant-Friedrichs-Lewy condition [96]. The code is usually solved in a $33 \times 16(r, z)$ point grid, for typical $1000-4000$ time steps within each rf period. The choice of finer grids leads to a severe degradation in the calculation time, with minor changes in the results. In general, $500-1000$ rf cycles are needed to meet the global convergence criterion: relative changes between two consecutive periods, below (i) $5 \times 10^{-4}$ for the charged particle densities, the electron mean energy, the plasma potential and the self-bias voltage; (ii) $10^{-3}$ for the neutral particles, corresponding to calculation times of $1-3$ hrs using a Fortan compiler on a Intel-Xeon E5520 (2.26 GHz) CPU.

As a result, the model yields the $2 \mathrm{D}$ profiles of the charged particle densities $n_{\alpha}$ and fluxes $\vec{\Gamma}_{\alpha}$, of the electron mean energy $\varepsilon$ and flux $\vec{\Gamma}_{\varepsilon}$, and of the rf field $\vec{E}$ and potential $V$; the values of the self-bias voltage $V_{d c}$ and of the effective power coupled to the plasma $W_{\text {eff }}$; and the average densities $\overline{n_{k}}$ of $\mathrm{N}_{2}\left(\mathrm{X}^{1} \Sigma_{g}^{+}, \mathrm{v}=0-45\right)$ (yielding the vdf $), \mathrm{N}_{2}\left(\mathrm{~A}^{3} \Sigma_{u}^{+}, \mathrm{B}^{3} \Pi_{g}, \mathrm{C}^{3} \Pi_{u}, \mathrm{a}^{1} \Sigma_{u}^{-}, \mathrm{a}^{1} \Pi_{g}, \mathrm{w}^{1} \Delta_{u}, \mathrm{a}^{\prime \prime 1} \Sigma_{g}^{+}\right)$and $\mathrm{N}\left({ }^{4} \mathrm{~S},{ }^{2} \mathrm{D},{ }^{2} \mathrm{P}\right)$, satisfying the closure condition $\sum_{k} \overline{n_{k}}+\sum_{i} \overline{n_{i}}=N$.

\section{Results and discussion}

This section presents numerical and experimental results characterizing the operation of ccrf discharges in pure nitrogen, at $0.1-1$ mbar pressures and $2-30 \mathrm{~W}$ coupled powers (corresponding to applied rf voltages in the range $100-300 \mathrm{~V}$ ).

An overall good agreement (qualitative and often also quantitative) is found between simulations and measurements, which exhibit the same order of magnitude and the same kind of evolution trends as a function of the power coupled to the plasma, for various pressures. Moreover, the LATMOS and the GREMI reactors have similar behaviours (hence justifying a presentation here of selected results only, namely considering the kind of measurements made in each reactor), although their different dimensions (radii and specially inter-electrode distances) can explain some distinctions in the operation features.

Figure 4(a)-(b) presents the time-average axial profiles (at discharge axis) of $E_{z} / N$ and of $n_{e}, n_{\mathrm{N}_{2}^{+}}, n_{\mathrm{N}_{4}^{+}}$, respectively, calculated for the LATMOS reactor at various pressures. For the same reactor, at 1 mbar, figure 4(c)-(d) shows $2 \mathrm{D}(r, z)$ plots of the electron mean energy $\varepsilon$ and of the ionization rate by electron impact $n_{e} \nu_{I}$, respectively. 
An observation of figure 4(a) confirms that the thickness of the space-charge sheaths corresponds approximately to 2/3 of the interelectrode distance (see the regions where $E_{z} / N \neq 0$ ), and that this thickness decreases with an increase in the pressure due to a more limited electron transport. Although there is no net charge-separation in the central part of the discharge (hence justifying $E_{z} / N=0$ in this region), figure 4(b) reveals that the charge particle density profiles are quite diverse. In particular, the relative importance in the $\mathrm{N}_{2}^{+}$to $\mathrm{N}_{4}^{+}$ion densities is highly dependent on the pressure (at 1 mbar, for example, both ions have similar populations), but the $n_{\mathrm{N}_{2}^{+}}$axial profile is the one whose shape changes the most with variations in the pressure. These profiles are a consequence of the relationship between the populations of electrons, ions and neutrals, interlinked through the main charged-particle production / destruction mechanisms: (i) direct and stepwise electron-impact ionization [reactions (6) and (7), see table 1]; (ii) associative ionization involving the excited states $\mathrm{N}_{2}(\mathrm{~A})$ and $\mathrm{N}_{2}(\mathrm{a}$ ) [reactions (23) and (24)]; (iii) the ion conversion from $\mathrm{N}_{4}^{+}$to $\mathrm{N}_{2}^{+}$and the three-body ion conversion from $\mathrm{N}_{2}^{+}$to $\mathrm{N}_{4}^{+}$[reactions (25) and (26), respectively]; and (iv) the electron recombination with $\mathrm{N}_{2}^{+}$and $\mathrm{N}_{4}^{+}$ions [reactions (10) and (11), respectively]. Mechanisms (i) and (ii) are dominant and their relative importance depends on the pressure: the contribution of the associative ionization to the charged-particle production is $35 \%$ at 0.2 mbar and $10 \%$ at 1 mbar. The results show also that transport effects are less important for $\mathrm{N}_{2}^{+}$ than for $\mathrm{N}_{4}^{+}$, especially at 1 mbar (notice that $\mu_{N_{4}^{+}}$is about $20 \%$ higher than $\mu_{N_{2}^{+}}$, see section 3.1); consequently, the $\mathrm{N}_{2}^{+}$ion is lost at the same positions where it is created, and its density profile reflects that of the ionization rate by electron impact [its main production mechanism at 1 mbar, see figure 4(d)]. Figure 4(c) shows that the electron mean energy exhibits maximum values in the sheath regions, with the consequent enhancement of reactivity (i.e. production / destruction rates) in these regions [this is confirmed in figure 4(d) for the electron collisions mechanisms]. Notice that the maxima of the electron mean energy (within the space-charge sheaths) are displaced from those of the charged particle densities, which occur halfway between the electrodes due to transport [see figure 4(b)]. Notice further the exceptional magnitude of $\langle\varepsilon\rangle$ at the corner $(r=R, z=0)(\sim 12 \mathrm{eV}$ for these conditions), due to the very small gap between the driven electrode and the grounded grid.

Figure 5(a)-(b) presents the axial profile of radiative intensities with the $\operatorname{SPS}(0,0)$ band and the $811.5 \mathrm{~nm}$ atomic argon line, measured (along the discharge cross section) and calculated (applying a time- and radial- average) for the GREMI reactor at various pressures. This figure shows that the $2 \mathrm{D}$ model yields an axial distribution for both the nitrogen of the argon intensities in good agreement with spatially resolved spectroscopic measurements. The latter are difficult to control, not only due to the extreme dependence of the emitted light intensity with position but also because of the limitations in defining a precise reference frame between the electrodes. These difficulties can justify the differences observed in the positions of the calculated and measured intensity peaks, which in any case do not call into question the validity of models results. 
The self-bias voltage, measured and calculated for both reactors at various pressures, is depicted in figure $6(\mathrm{a})-(\mathrm{b})$ as a function of the power coupled to the plasma. Note that a increase in the coupled power and a decrease in the pressure, both lead to a more asymmetric discharge operation (hence to a higher self-bias voltage), due to an enhancement in the charged-particle fluxes towards the electrodes. A good agreement is found (both quantitative and qualitative) between $V_{\mathrm{dc}}$ simulations and measurements for the LATMOS reactor. For the GREMI reactor, the model predicts the correct evolution trend for $V_{\mathrm{dc}}$ with variations in both $W_{\text {eff }}$ and $p$, yet yielding values that seem systematically deviated with respect to the experiment, which might be due to uncertainties in the wall loss probabilities adopted. In any case, the quality of the agreement for a global parameter such as the self-bias voltage, obtained using two different experimental setups, constitutes another successful test for model validation.

Figure 7 presents the space-time average electron density, as a function of the power coupled to the plasma, measured and calculated for the GREMI reactor at various pressures. As for $V_{\mathrm{dc}}$, one observes $\left\langle n_{e}\right\rangle$ to increase with $W_{\text {eff }}$ (at constant pressure) simply due to the enhanced energy involved in plasma maintenance. Notice that a decrease in $p$ (at constant power coupled) leads to an increase in $\left\langle n_{e}\right\rangle$ (for most conditions), which is associated with an increase also in $\varepsilon$, probably to compensate for higher (particle and energy) losses. Ultimately, this variation is responsible for a significant increase in the ionization degree, when $p$ decreases at constant $W_{\text {eff }}$. Figure 7 shows that the calculated electron density is underestimated by a factor of about 4 with respect to experiment, which is a typical limitation of fluid simulations when applied to the modelling of ccrf discharges. This is probably associated with the fact that the electron flux equation $(2 b)$ neglects the corresponding inertia term, which overestimates the energy budget required to maintain the rf sheaths leaving less energy available for the electron production. However, even with this limitation, the model is capable to provide correct qualitative predictions for the evolution of $\left\langle n_{e}\right\rangle$ with $W_{\text {eff }}$ and $p$.

Figure 8(a)-(c) presents the space-time average radiative intensities, as a function of the power coupled to the plasma, of the $\operatorname{SPS}(0,2)$ and the $\operatorname{FNS}(0,0)$ bands, and of the $811.5 \mathrm{~nm}$ atomic argon line, measured and calculated for the LATMOS reactor at various pressures. Measurements were carried out in the vicinity of the driven electrode, where the emitted light intensity is maximum, and thus they are compared with spatiallyaveraged simulations results, calculated over the entire discharge cross section (in the radial direction) and over $1 / 3$ of the interelectrode distance (in the axial direction, below the driven electrode). The experimental observations reveal that the transition intensities are highly dependent on position (within $\mathrm{mm}$, see figure 5), which makes extremely difficult to control the measurement of spatially-averaged data to compare with the simulations. Within each figure 8(a)-(c) the model results are normalized to a single experimental point, because no absolute calibration of the optical device has been done and thus the OES data are in arbitrary units. A fair agreement is found between simulations and measurements, particularly at low pressures and low coupled powers. Figure $8(\mathrm{~b})$ shows that the evolution of the $\operatorname{FNS}(0,0)$ intensity, with variations in either 
the pressure or the coupled power, is similar to that of the electron density [see figure 7 and the inset in figure 8(b)], thus confirming the relevant role of the electron collisions in the production of $\mathrm{N}_{2}^{+}$and the coherency of the present study (the $n_{e}$ measurements were made in the GREMI reactor, whereas the spatially-averaged OES measurements were performed in the LATMOS reactor). Note that the $\operatorname{SPS}(0,2)$ and the $\operatorname{Ar}(811 \mathrm{~nm})$ intensities exhibit different behaviours with changes in $p$ and $W_{\text {eff }}$. The argon line follows the same variation trends as $n_{e}$ (decreasing with an increase in $p$ and increasing with $\left.W_{\text {eff }}\right)$, whereas the $\operatorname{SPS}(0,2)$ band increases with $p$ and tends to saturate as $W_{\text {eff }}$ increases. These differences can be explained by a combination of factors: (i) the argon excitation is assumed to proceed via direct electron collisions only, whereas the $\mathrm{N}_{2}(\mathrm{C})$ excited state is create not only by the direct electron excitation (4a) but also by the pooling reaction (15b) (see Table 1), whose contribution to the total $\mathrm{N}_{2}(\mathrm{C})$ production is $5 \%$ at 1 mbar and $26 \%$ at 0.2 mbar; (ii) the differences, both in threshold and in shape, between the electron-impact excitation cross sections of $\mathrm{N}_{2}(\mathrm{C})$ and of Ar lead to different spatial distributions of the corresponding time-average electron rate coefficients, hence to different local production rates with these mechanisms whose values, in any case, decrease with the pressure.

Figure 9(a)-(b) shows the time-average electron energy probability function $f(u) u^{1 / 2}$ as a function of $u$, calculated for the LATMOS reactor at $p=1$ mbar and $W_{\text {eff }}=7.4 \mathrm{~W}$ and at $p=0.5$ mbar and $W_{\text {eff }}=5 \mathrm{~W}$ (corresponding to $V_{\mathrm{rf}} \simeq 200 \mathrm{~V}$ ). By using the local mean energy approximation (see section 3.1) the eedf becomes spatially resolved via its dependence with $\varepsilon$ and $n_{e}$ [see equation (15)], and thus results are obtained in the discharge centre $(r=0$ and $z=16.5 \mathrm{~mm})$ and in the space-charge sheath with the driven electrode $(r=0$ and $z=4.1 \mathrm{~mm})$, where the OES measurements were done (see figure 8). Figure 9 reveals that a change in the pressure induces a considerable modification of the eedf in the space-charge sheath, but the same is not observed in the plasma bulk where the eedf's shape is not altered by pressure variations. In both regions a decrease in $p$ yields an increase in the tail of $f(u) u^{1 / 2}$ and a simultaneous decrease in its body, but these modifications are particularly significant in the sheath where $\varepsilon$ changes from 5.9 to $9.7 \mathrm{eV}$ when $p$ goes from 1 to 0.5 mbar. Notice that the increase in the eedf's tail with the decrease in the pressure, although implying an enhancement in the electron collision rate coefficients, does not augment the relative contribution of the electron impact mechanisms for charged particle production, at low pressures. This (apparent) contradiction is associated with the extraordinary increase observed in the $\mathrm{N}_{2}(\mathrm{~A})$ and $\mathrm{N}_{2}\left(\mathrm{a}^{\prime}\right)$ populations when the pressure decreases [see figure 10(a)-(b)].

Figure 10(a)-(b) presents the relative densities of the $\mathrm{N}_{2}(\mathrm{~A})$ and the $\mathrm{N}_{2}\left(\mathrm{a}^{\prime}\right)$ metastable states, as a function of the power coupled to the plasma, calculated for the LATMOS reactor at various pressures. These species play an important role in the electron production [via the associative ionization reactions (23) and (24), see table 1] and in the population of the $\mathrm{N}_{2}(\mathrm{C})$ state responsible for the SPS transition [via the pooling reaction (15b), see table 1]. The densities $n_{\mathrm{N}_{2}(\mathrm{~A})} / N$ and $n_{\mathrm{N}_{2}\left(\mathrm{a}^{\prime}\right)} / N$ become larger with either a decrease in $p$ or an increase in $W_{\text {eff }}$ (particularly at low pressures), due to 
the increase in both $n_{e}$ and $\varepsilon$. Incidentally, the same kind of variation was reported about measurements of the $\mathrm{N}_{2}($ A) density in inductively coupled plasmas (at lower pressures and higher coupled powers) [97]. Notice that the main production mechanisms of states $\mathrm{N}_{2}(\mathrm{~A})$ and $\mathrm{N}_{2}\left(\mathrm{a}\right.$ ') proceed, respectively, via the states $\mathrm{N}_{2}(\mathrm{~B})$ [pooling reaction (16a) and radiative transition (28)] and $\mathrm{N}_{2}(\mathrm{a})$ [pooling reaction (18) and electron excitation (4a)], which are created via collisions with $\mathrm{N}_{2}(\mathrm{~A})$ itself [vibrational de-excitation (14)] and with electrons [reaction (4a)]. The vibrational excited states $\mathrm{N}_{2}(\mathrm{X}, \mathrm{v}=2-10)$ are also populated essentially by direct electron excitation. Ultimately, the electron kinetics controls the population of the metastables, justifying their variations with the working conditions.

Figure 11 plots the dissociation degree of nitrogen $n_{\mathrm{N}} /\left(n_{\mathrm{N}_{2}}+n_{\mathrm{N}}\right)$, as a function of the power coupled to the plasma, calculated for the LATMOS reactor at various pressures. One observes weak dissociation degrees, varying between $10^{-3}$ and $4 \times 10^{-2}$ for increasing $W_{\text {eff }}$ values (at standard $\gamma_{\mathrm{N}_{2}}^{\prime}=4.5 \times 10^{-4}$ and $\gamma_{\mathrm{N}}^{\prime}=10^{-3}$ conditions) and exhibiting little changes with $p$. The results displayed show also that the dissociation degree decreases with an increase in the wall loss probabilities for atoms (as expected, a significant effect is observed) and vibrationally excited molecules, $\gamma_{N}^{\prime}$ and $\gamma_{\mathrm{N}_{2}}^{\prime}$, respectively. These results can be explained by noticing that the main dissociation mechanisms of nitrogen involve collisions with highly excited vibrational states [above $\mathrm{v}>10$, see reactions (22) and (41) in tables 1 and 2], whose kinetics is essentially governed by $\mathrm{N}_{2}-\mathrm{N}_{2} \mathrm{~V}-\mathrm{V}$ and $\mathrm{N}_{2}-\mathrm{N}$ V-T mechanisms [reactions (38) and (13), respectively] that are favoured by an increase in both the coupled power and the pressure. Consequently, the $\mathrm{N}(\mathrm{S}, \mathrm{D}, \mathrm{P})$ populations are roughly proportional to $p$ and the dissociation degree presented in figure 11 displays negligible variations with the pressure. This is in contrast with the results of figure 7 , which suggest an important variation of the ionization degree with the pressure (in coherency with the evolution of $n_{\mathrm{N}_{2}(\mathrm{~A})} / N$ and $n_{\mathrm{N}_{2}\left(\mathrm{a}^{\prime}\right)} / N$ vs. $p$ plotted in figure 10 ), regardless the fact that the ionization energy is higher than the dissociation energy in nitrogen. This apparent contradiction puts forward the existence of different mechanisms, associated with the electron and the vibrational kinetics, in control of the ionization and the dissociation of nitrogen, respectively.

As mentioned in section 3.2, the kinetic scheme presented in Tables 1-2 depends on several parameters $\left(b_{\text {ion }}, b_{\text {atom }}, \gamma_{\mathrm{N}}, \gamma_{\mathrm{N}_{2}}\right.$, and $\left.\xi_{e}\right)$, whose values can influence the model results. Simulations tests show that: (i) the branching ratios $b_{\text {ion }}$ and $b_{\text {atom }}$ can vary between $0-1$ with little influence upon the results; (ii) the wall atomic recombination probability has an (obvious) direct effect upon the dissociation degree, which decreases by a factor of $\sim 10^{2}$ when $\gamma_{\mathrm{N}}^{\prime}$ increases from $10^{-3}$ to 1 . This variation in $\gamma_{\mathrm{N}}^{\prime}$ yields also a maximum increase of $\sim 12 \%$ in $n_{e}$, due to a reduction in the destruction of the metastable $\mathrm{N}_{2}(\mathrm{~A})$ by atomic impact (recall that the associative ionization involving $\mathrm{N}_{2}(\mathrm{~A})$ is one of the main electron production channels); (iii) an increase in the wall vibrational deeexcitation probability reduces significantly the population of the lower vibrationally excited states, thereby affecting the shape of the vdf. This has a direct 
impact upon the dissociation degree (controlled by the vibrational kinetics) and the electron production (controlled by direct / stepwise electron impact ionization, which is favoured by the quenching of vibrationally excited states down to the groundstate). The dissociation degree and the electron density decrease $\sim 93 \%$ and increase $\sim 45 \%$, respectively, when $\gamma_{\mathrm{N}_{2}}^{\prime}$ varies from $4.5 \times 10^{-4}$ to 1 at $0.5 \mathrm{mbar}$ and $10 \mathrm{~W}$; (iv) variations in the secondary electron emission coefficient produce negligible changes in the electron density, although affecting the self-bias voltage through changes in the boundary condition $(7 a)$. By increasing $\xi_{e}$ from 0 to $0.5,\left|V_{\mathrm{dc}}\right|$ reduces $\sim 10 \%$ at 0.5 mbar and $10 \mathrm{~W}$.

\section{Final remarks}

This paper has studied ccrf plasma discharges in pure nitrogen, using both experiments and simulations. Experiments obtained relevant parameters (the rf-applied and the dc-self-bias voltages, the effective power coupled to the plasma, the average electron density, and the intensities of radiative transitions with the nitrogen second-positive and first-negative systems and with the $811.5 \mathrm{~nm}$ atomic line of argon), recorded in the LATMOS and the GREMI laboratories. Measurements were made independently and with different equipment upon two similar (not twin) experimental setups (cylindrical parallel-plate reactors surrounded by a lateral grounded grid), at $p=0.1-1$ mbar pressures and $W_{\text {eff }}=2-30 \mathrm{~W}$ effective powers coupled to the plasma (corresponding to applied rf voltages $V_{\text {rf }}=100-300 \mathrm{~V}$ ). The coupled powers were measured using the subtractive method (with and without plasma), thus accounting for the power losses in the external circuitry (the matchbox, the coaxial cables and the different connectors). Simulations used a hybrid code that couples a 2D $(r, z)$ time-dependent fluid module to a very complete $0 \mathrm{D}$ kinetic module. The fluid module solves the continuity and the momentum-transfer equations for electrons and positive ions $\mathrm{N}_{2}^{+}(\mathrm{X}), \mathrm{N}_{2}^{+}(\mathrm{B})$ and $\mathrm{N}_{4}^{+}$, the electron mean energy transport equations, and Poisson's equation for the rf electric potential. The kinetic module solves the two-term homogeneous and stationary electron Boltzmann equation (accounting for inelastic collisions from ground-state molecules and atoms, and inelastic and superelastic collisions involving vibrationally excited states) and the rate balance equations of 45 vibrational excited states with the ground-state $\mathrm{N}_{2}\left(\mathrm{X}^{1} \Sigma_{g}^{+}, \mathrm{v}=0-45\right), 7$ electronic states $\mathrm{N}_{2}\left(\mathrm{~A}^{3} \Sigma_{u}^{+}, \mathrm{B}^{3} \Pi_{g}, \mathrm{C}^{3} \Pi_{u}, \mathrm{a}^{\prime 1} \Sigma_{u}^{-}, \mathrm{a}^{1} \Pi_{g}, \mathrm{w}^{1} \Delta_{u}\right.$, $\left.\mathrm{a}^{\prime \prime} \Sigma_{g}^{+}\right)$with the nitrogen molecule, and 3 electronic states $\mathrm{N}\left({ }^{4} \mathrm{~S},{ }^{2} \mathrm{D},{ }^{2} \mathrm{P}\right)$ with nitrogen atoms.

The code was validated by comparison between simulations and measurements, yielding a good agreement (within the experimental uncertainties) for the self-bias voltage and for the intensities of radiative transitions (both average and spatiallyresolved), at different pressures and coupled powers. This validation showed that it is possible to join a sophisticated 2D space-time dependent discharge code to a complex kinetic scheme to model ccrf discharges in pure nitrogen. Moreover, the validation used experimental results obtained from two separate setups, which corresponds to 
exceptional benchmarking conditions not only for this work but also for future works. Finally, because model validation has been based also on the analysis of optical emission spectroscopy diagnostics, it further allowed to clarify the mechanisms controlling the kinetics of the main excited species with nitrogen. In particular, experimental SPS results are only matched if the $\mathrm{N}_{2}(\mathrm{~A})$ density, $n_{e}$ and the electrical parameters are correct, whereas the FNS emission is only properly modelled if both the calculated $\mathrm{N}_{2}^{+}$ density and the vdf are accurate. Notice that we have chosen here to describe the evolution of the relevant physical quantities as a function of $W_{\text {eff }}$ instead of $V_{\mathrm{rf}}$. This choice is because, in the present model, neither the inertial terms in the electron flux equation (2b) were accounted for, nor the external power-circuit was considered in a self-consistent way, which can alter the phase between $V(t)$ and $I_{t}(t)$, and thus the relationship between $V_{\text {rf }}$ and $W_{\text {eff }}$.

Results exhibit a strong spatial non-homogeneity that depends particularly on the operating pressure. These features are well predicted by the $2 \mathrm{D}$ transport effects considered in the model, which adopts the local mean energy approximation to define space-time dependent electron parameters for the fluid module and to work-out spacetime average rates for the kinetic module. The success of this spatial description depends mostly on the calculated eedf, whose tail is more populated in the space-charge sheath region than in the plasma bulk region, especially at low pressure. Transport effects justify also that the maxima of the charged particle densities occurs at the reactor centre, displaced from the maxima of their production rates (located within the spacecharge sheaths). These results show the need to adopt the complete $2 \mathrm{D}$ space-time description considered here to provide an adequate modelling of these discharges.

Simulations tests show that the model predictions are not significantly affected (variations below 10\%) by changes in (i) the branching ratios for the production of atoms and of ions, (ii) the secondary electron emission coefficient, (iii) the frequency adopted for updating the nitrogen kinetics in calculations and (iv) the profiles imposed in the model for the molecular and the atomic excited species. As expected, the probabilities for the atomic recombination and the vibrational deeexcitation at the wall have a direct effect upon both the dissociation degree and the electron density. However, even by setting these probabilities to unity the dissociation degree remains low and $n_{e}$ increases not more than $45 \%$. This shows that the highly-complex kinetic scheme proposed here is able to capture the main plasma features (within experimental errors), even considering the uncertainties associated with some of the collisional data.

As usually observed in fluid models applied to ccrf discharges, simulations underestimate the electron density by a factor of $3-4$, yet yielding correct qualitative predictions for the evolution of $\left\langle n_{e}\right\rangle$ with $W_{\text {eff }}$ and $p$. Electrons are produced mainly by direct and stepwise electron-impact ionization and by associative ionization involving the metastables states $\mathrm{N}_{2}(\mathrm{~A})$ and $\mathrm{N}_{2}\left(\mathrm{a}^{\prime}\right)$; these mechanisms yield a relatively low electron density $\left(\left\langle n_{e}\right\rangle \simeq 5 \times 10^{8}-5 \times 10^{9} \mathrm{~cm}^{-3}\right)$ due to the limited coupled-power characterizing these discharges. At $10 \mathrm{~W}$ the ionization degree ranges from $6 \times 10^{-8}$ at 1 mbar to $4 \times 10^{-7}$ at 0.5 mbar, and this variation is accompanied by an increase in the populations of $\mathrm{N}_{2}(\mathrm{~A})$ 
and $\mathrm{N}_{2}\left(\mathrm{a}^{\prime}\right)$. Even if the ionization of nitrogen is essentially controlled by the electron kinetics, its dissociation is governed by the vibrational kinetics and by the atomic losses at the walls. The dissociation degree is very weak, varying between $10^{-3}$ and $4 \times 10^{-2}$ for increasing coupled powers (these are maximum values, obtained for a vanishingly small atomic wall-loss probability), being associated with atomic populations that are roughly proportional to the pressure. Since the populations of atoms are not easily measurable, this is an important model result. The reliability of the predictions for the dissociation degree is indirectly ensured by the good agreement between simulations and measurements for the intensities of radiative transitions, since both results depend on the accuracy of the kinetic description for the excited $\mathrm{N}_{2}$ and $\mathrm{N}$ species.

The present work is part of a more extended study aiming characterizing ccrf discharge plasmas produced in nitrogen-methane mixtures (for $\mathrm{CH}_{4}$ concentrations up to $10 \%$ ), for the laboratory synthesis of analogues to Titan's solid aerosols. The step-bystep methodology adopted was initiated with the study of pure $\mathrm{N}_{2}$ discharges presented here, which allowed to set well-grounded physical basis for understanding these plasmas (in terms of the discharge production features, the transport description adopted, and the kinetic mechanisms considered), before moving to more complex situations. This strategy will continue with the study of $\mathrm{N}_{2}-\mathrm{H}_{2}$ and of $\mathrm{N}_{2}-\mathrm{CH}_{4}$ ccrf discharges, the latter including dust formation.

\section{Acknowledgments}

Work supported by a PICS Cooperation Program, financed by the Portuguese Foundation for Science and Technology (FCT) and by the Centre National de la Recherche Scientifique (CNRS). The calculations were performed on SeARCH (Services \& Advanced Computing with HTC/HPC) funded by FEDER through the COMPETE program and by the Portuguese FCT under contract CONC-REEQ/443/EEI/2005. Et Es-sebbar thanks the ANR programme (ANR-09-JCJC-0038 contract) for his PostDoctoral grant. 
Figure captions

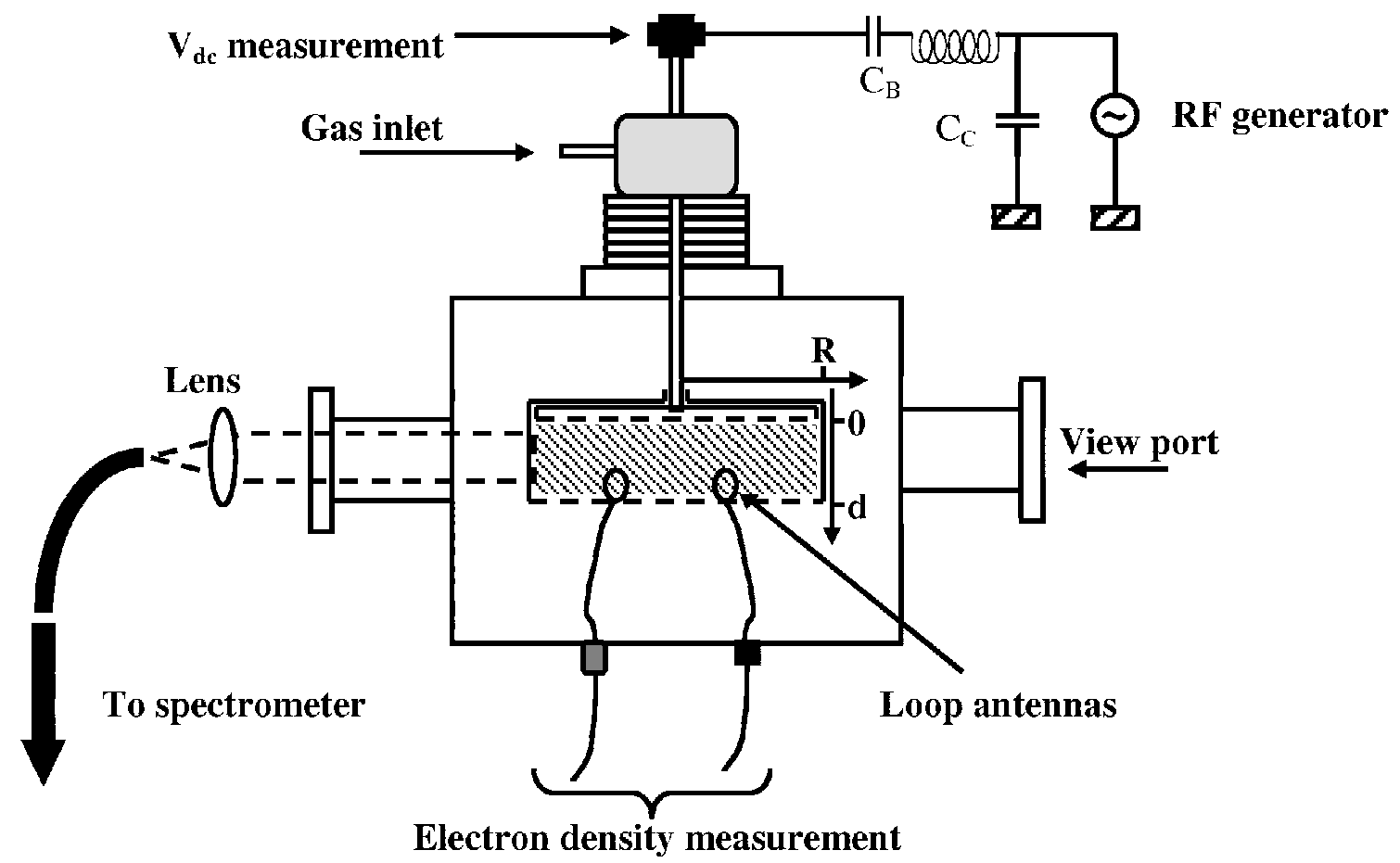

Figure 1. Schematic diagram of the plasma chamber and the external circuit with the capacitively coupled reactors used in this work. The plasma is the shaded region, between the driven (top) and the grounded electrodes. 


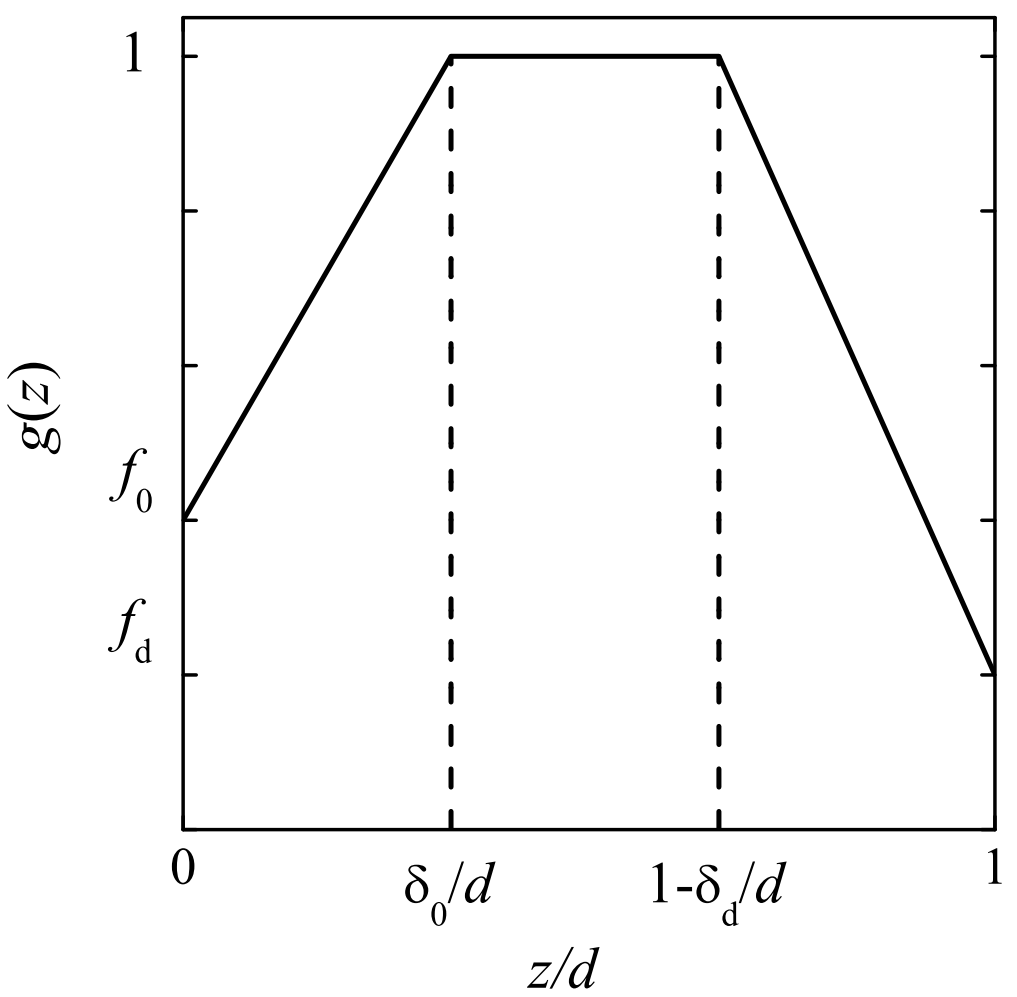

Figure 2. Axial density profile $g(z)$ [spatially homogeneous in the plasma bulk and linearly decreasing in the plasma sheaths, see equation $(22 b)]$, adopted in this work for $\mathrm{N}_{2}\left(\mathrm{~A}^{3} \Sigma_{u}^{+}, \mathrm{a}^{1} \Sigma_{u}^{-}, \mathrm{a}^{1} \Pi_{g}, \mathrm{w}^{1} \Delta_{u}\right)$ and $\mathrm{N}\left({ }^{2} \mathrm{D},{ }^{2} \mathrm{P}\right)$. In this figure, $\delta_{0}$ and $\delta_{d}$ are the sheath thickness near the driven electrode and the grounded electrode, respectively; $f_{0}$ and $f_{d}$ are the ratio of the wall density to the bulk density calculated at $z=0$ and $z=d$, respectively. The radial profile follows equation $(22 c)$ and is similar to this one. 


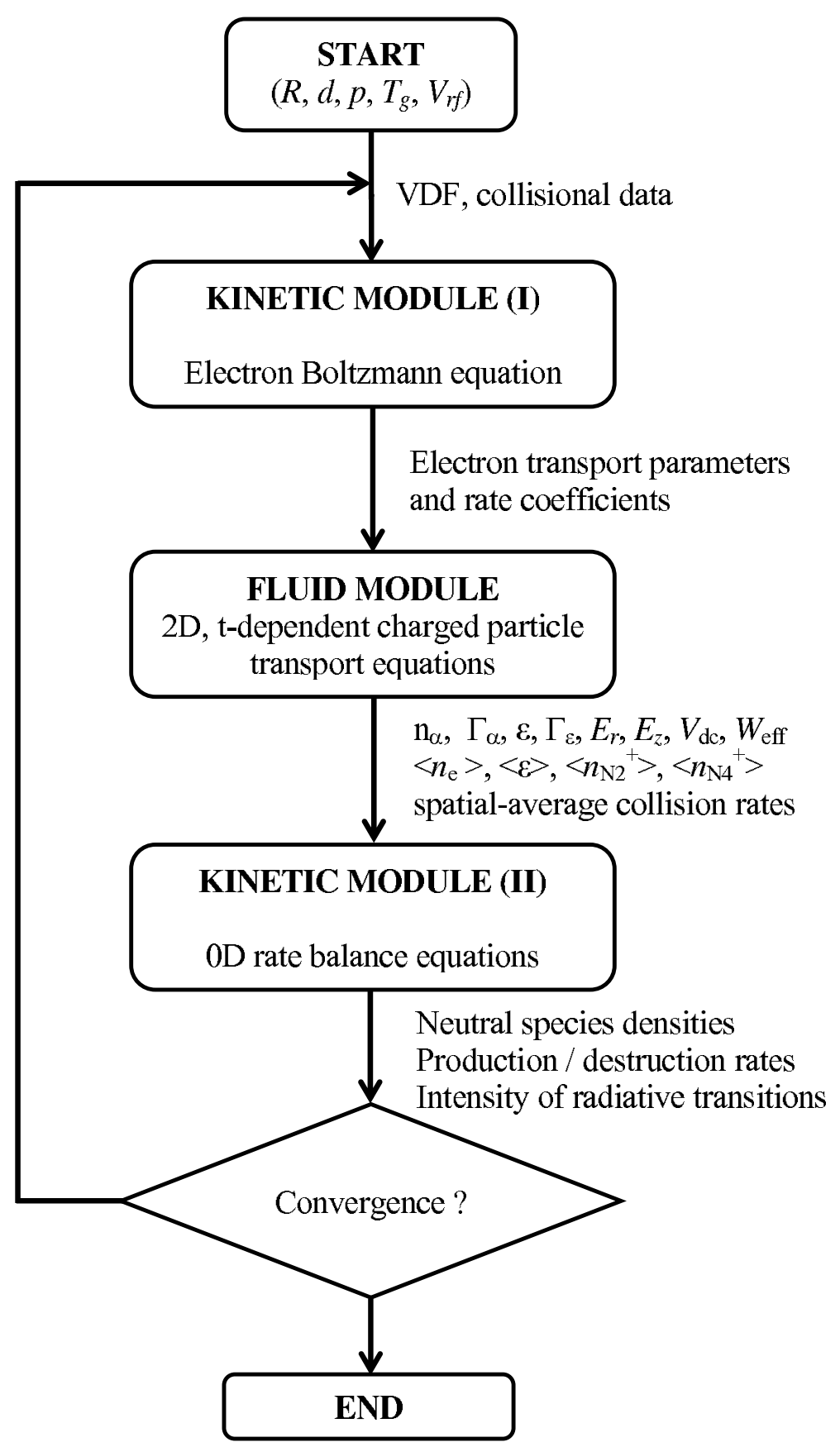

Figure 3. Flowchart of the numerical workflow used in the simulations. 

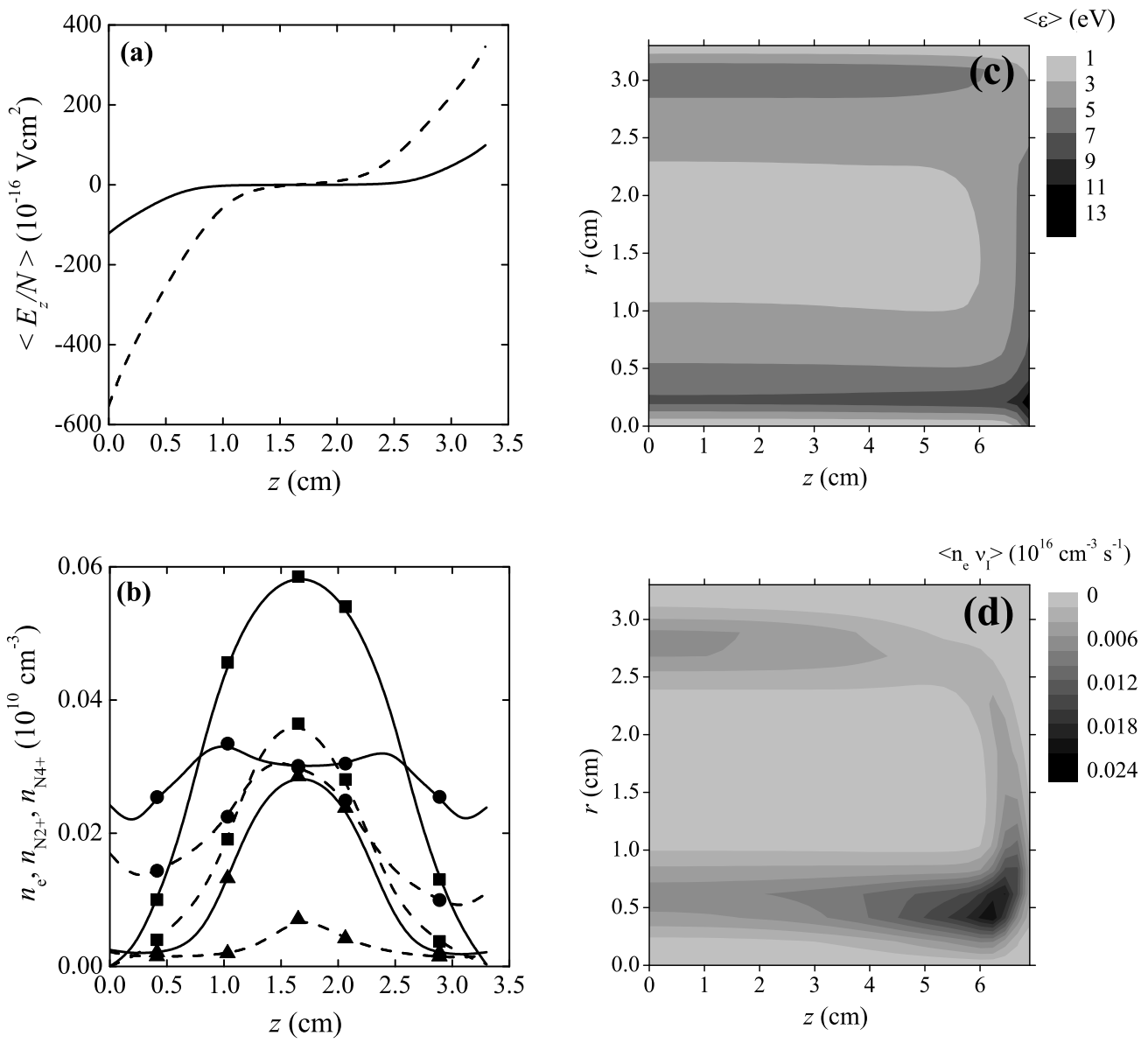

Figure 4. Calculated time-average profiles (for the LATMOS reactor) of the following quantities: (a) the axial reduced electric field and (b) the densities of electrons $(\mathbf{\square}), \mathrm{N}_{2}^{+}$ $(\boldsymbol{O})$ and $\mathrm{N}_{4}^{+}(\boldsymbol{\Delta})$ ions, as a function of $z$ at discharge axis $(r=0)$, for $p=1 \mathrm{mbar}$ (solid line) and $p=0.2$ mbar (dashed); (c) the electron mean energy and (d) the ionization rate by electron impact, as a function of $r$ and $z$ for $p=1$ mbar pressure. The power coupled to the plasma is $7.4 \mathrm{~W}$ at $1 \mathrm{mbar}$ and $2.3 \mathrm{~W}$ at $0.2 \mathrm{mbar}$, corresponding to $V_{\text {rf }} \simeq 200 \mathrm{~V}$. 

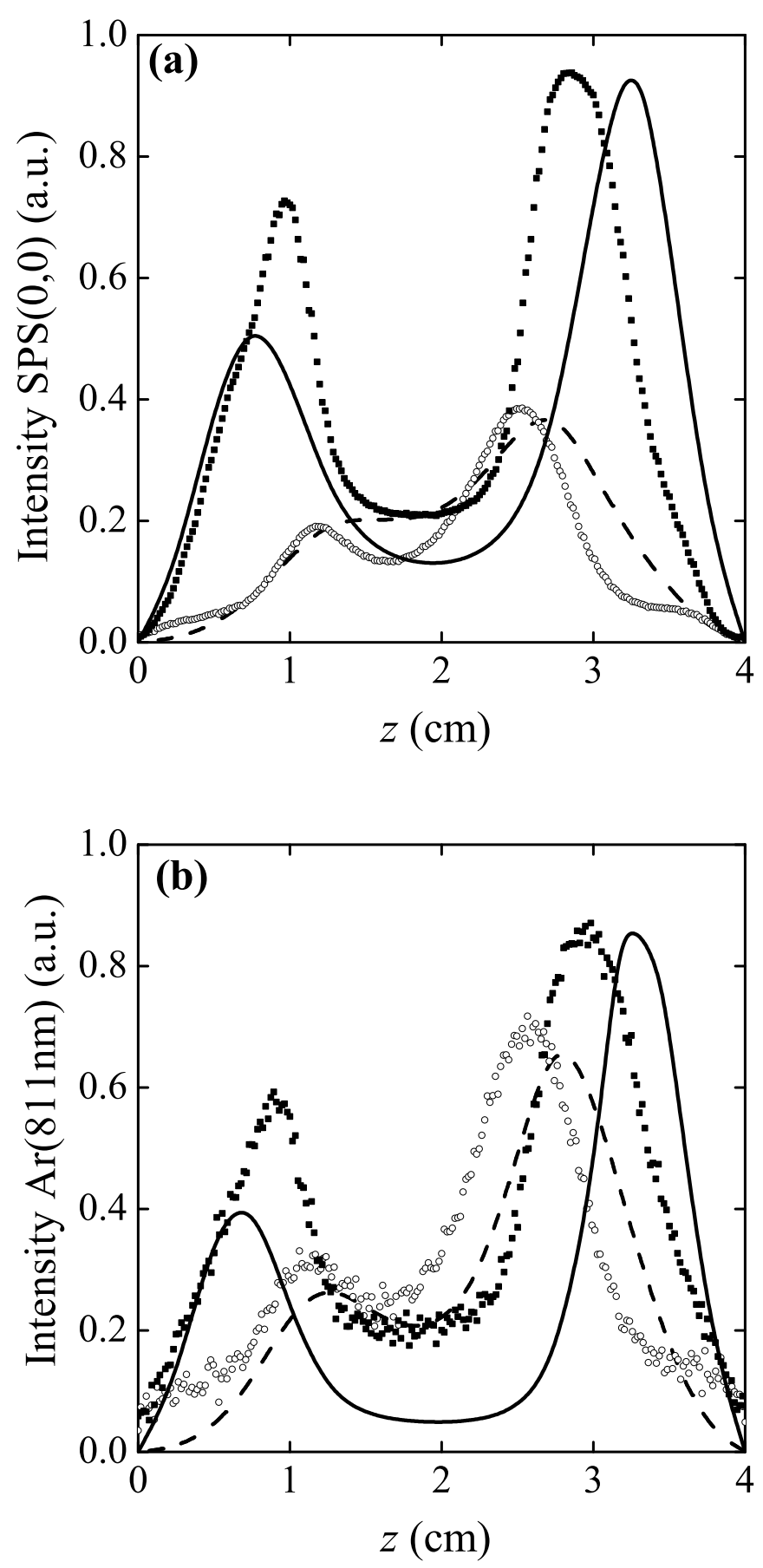

Figure 5. Time- and radial- average radiative intensities of the $\operatorname{SPS}(0,0)$ band (a) and the $811.5 \mathrm{~nm}$ atomic argon line (b), measured (points) and calculated (lines) between the electrodes of the GREMI reactor, for the following work conditions (at $V_{\mathrm{rf}} \simeq 145 \mathrm{~V}$ ): $p=1$ mbar and $W_{\text {eff }}=4 \mathrm{~W}$ (solid line and $\boldsymbol{\square}$ ); $p=0.2$ mbar and $W_{\text {eff }}=16 \mathrm{~W}$ (dashed and $\mathrm{O}$ ). For each condition, the maxima of the experimental and the calculated curves were normalized to the same value. 

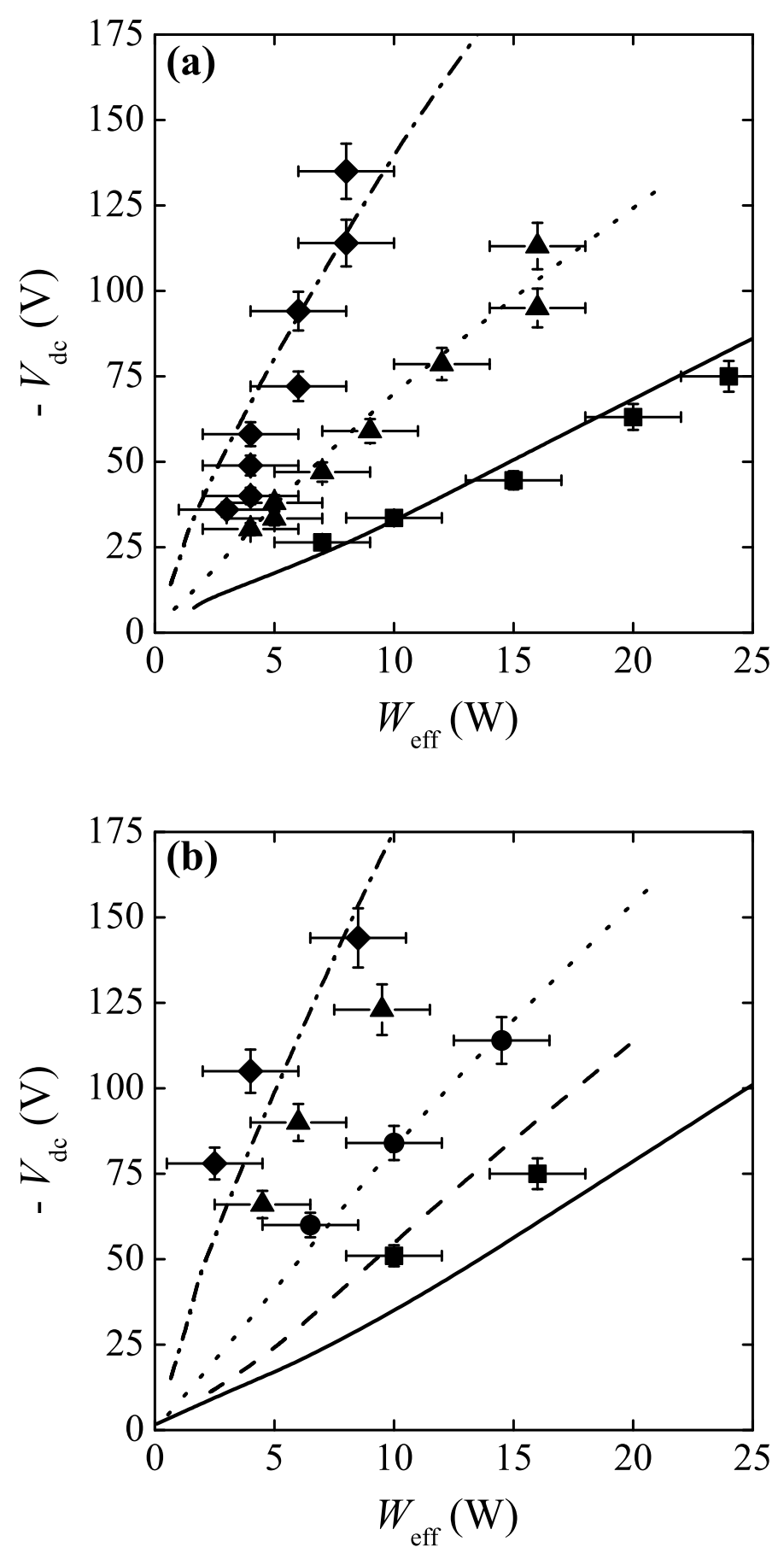

Figure 6. Self-bias voltage, as a function of the power coupled to the plasma, measured (points) and calculated (lines) in the LATMOS (a) and the GREMI (b) reactors, for the following pressures (in mbar): 1 (solid line and $\boldsymbol{\square}$ ); 0.72 (dashed and $\bullet$ ); 0.5 (dotted and $\mathbf{\Lambda}$ ); 0.2 (dashed-dotted and $\$$ ). 


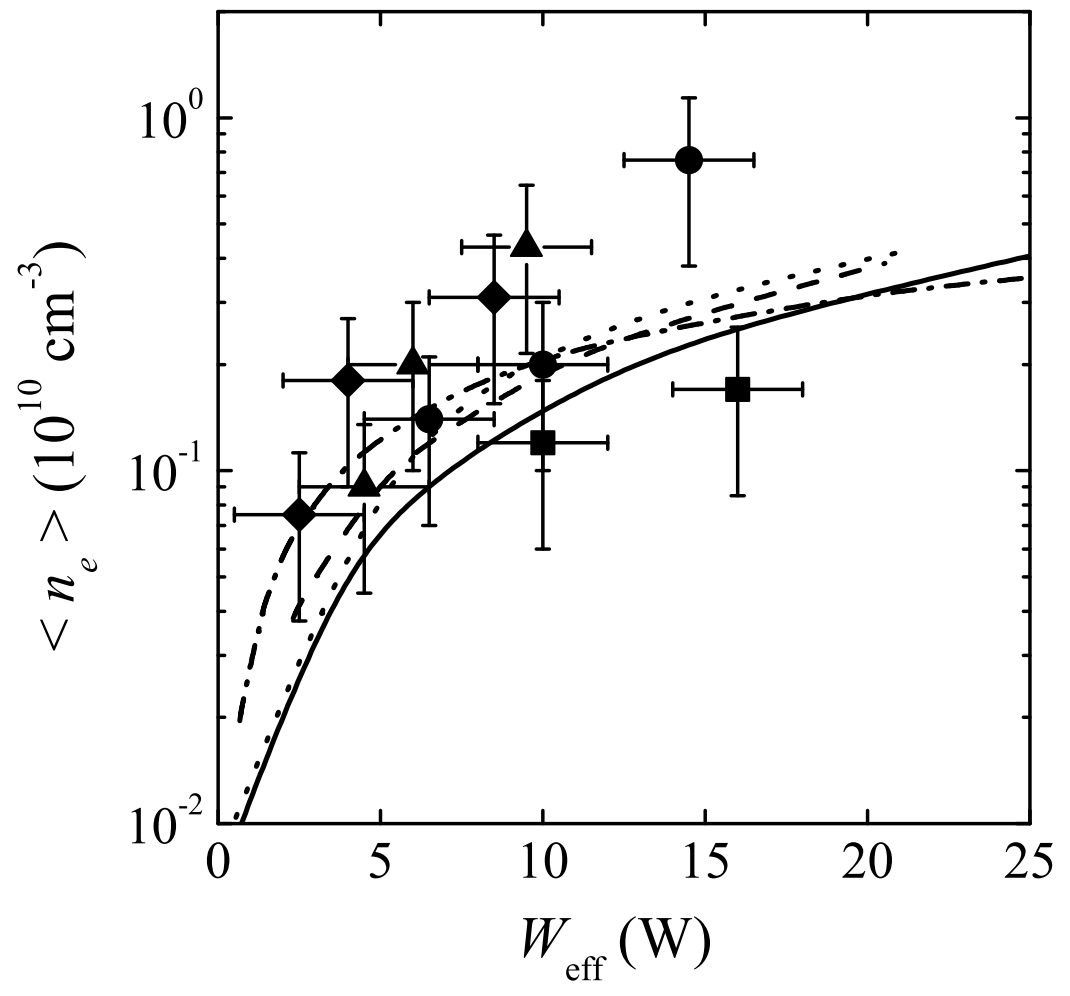

Figure 7. Space-time average electron density, as a function of the power coupled to the plasma, measured (points) and calculated (lines) in the GREMI reactor, for the following pressures (in mbar): 1 (solid line and $\mathbf{\square}$ ); 0.72 (dashed and $\boldsymbol{0}$ ); 0.5 (dotted and $\boldsymbol{\Delta}$ ); 0.2 (dashed-dotted and $\diamond$ ). Simulations are multiplied by a factor of 4 , for representation purposes. 

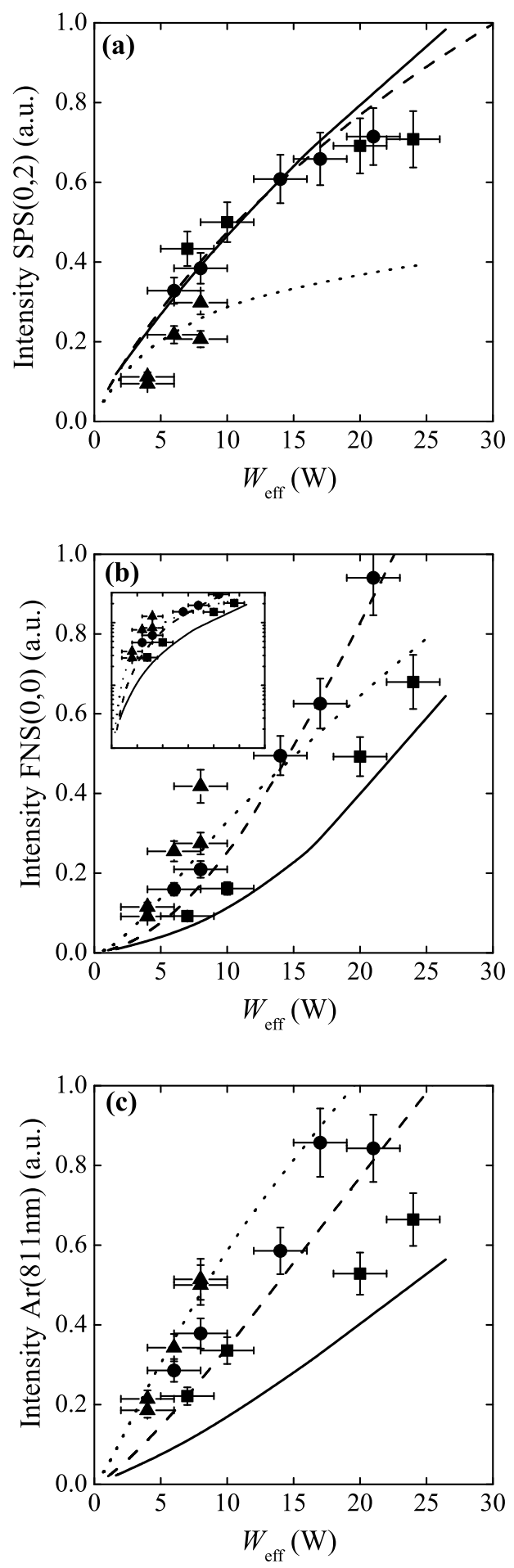

Figure 8. Space-time average radiative intensities, as a function of the power coupled to the plasma, of the $\operatorname{SPS}(0,2)$ band (a), the $\operatorname{FNS}(0,0)$ band (b), and the $811.5 \mathrm{~nm}$ atomic argon line (c), measured (points) and calculated (lines) in the LATMOS reactor, for the following pressures (in mbar): 1 (solid line and $\boldsymbol{\square}$ ); 0.6 (dashed and $\bullet$ ); 0.2 (dotted and $\mathbf{\Delta}$ ). The inset in figure 8 (b) is just its semilog representation. Within each subfigure (a),(b) and (c), all simulation values are normalized to one experimental point obtained at: $\sim 7 \mathrm{~W}$ and 0.6 mbar for the $\operatorname{SPS}(0,2) ; \sim 7 \mathrm{~W}$ and $0.2 \mathrm{mbar}$ for the $\operatorname{FNS}(0,0) ; \sim 7 \mathrm{~W}$ and 0.2 mbar for the $\operatorname{Ar}(811 \mathrm{~nm})$. Simulations were averaged radially, over the entire discharge cross section, and axially, over $1 / 3$ of the interelectrode distance below the driven electrode (corresponding to the region where the OES measurements were carried out). 

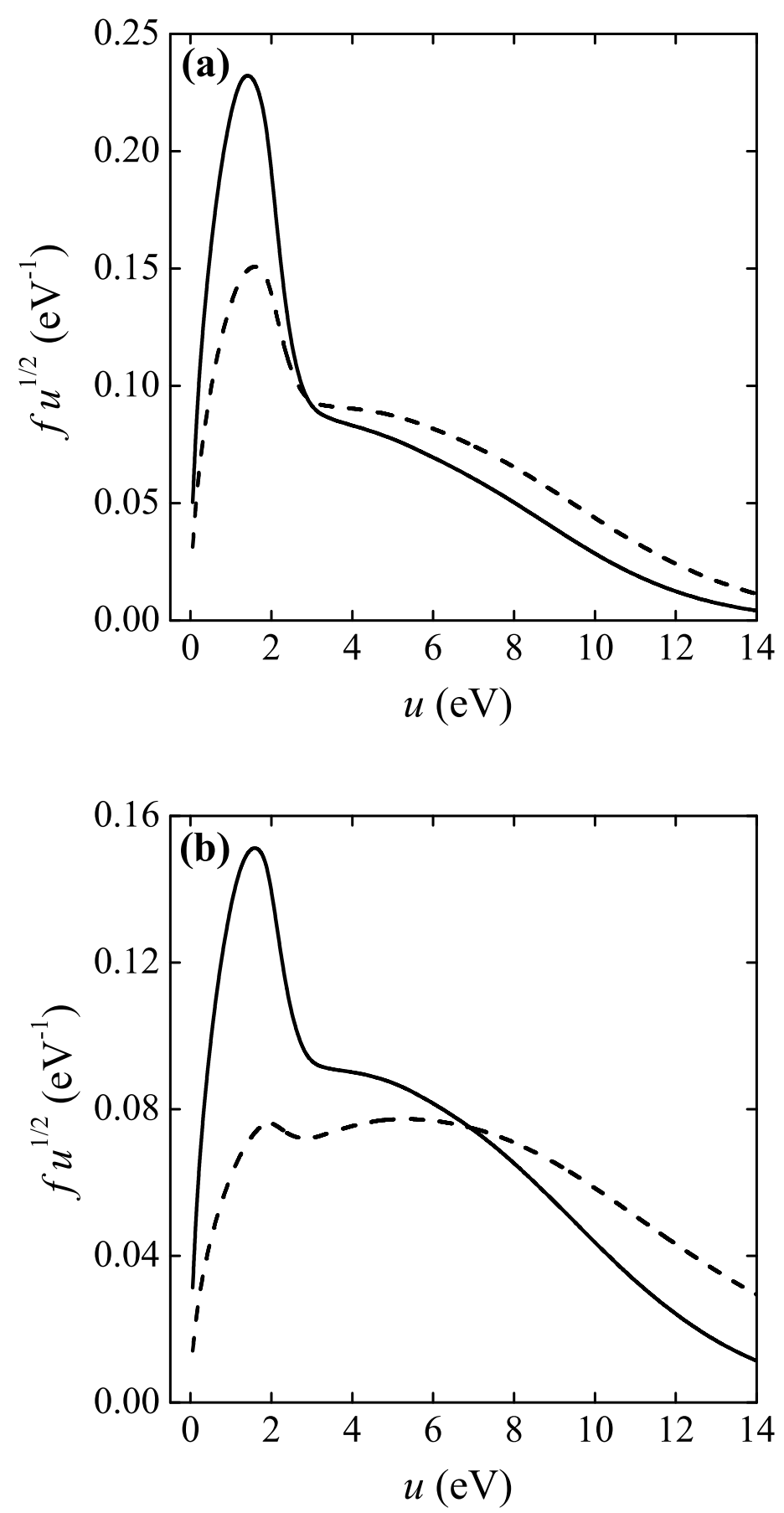

Figure 9. Time-average electron energy probability function $f(u)_{\mid\langle\varepsilon\rangle,\left\langle n_{e}\right\rangle} u^{1 / 2}$, as a function of the kinetic energy $u$, calculated for the LATMOS reactor and the following work conditions (at $\left.V_{\mathrm{rf}} \simeq 200 \mathrm{~V}\right): p=1 \mathrm{mbar}$ and $W_{\text {eff }}=7.4 \mathrm{~W}$ (solid lines); $p=0.5 \mathrm{mbar}$ and $W_{\text {eff }}=5 \mathrm{~W}$ (dashed). The results correspond to: (a) the discharge centre (at $r=0$ and $z=16.5 \mathrm{~mm}$ ), where the time-average electron mean energy is $\langle\varepsilon\rangle \simeq 2.4$ and $2.7 \mathrm{eV}$ for $p=1$ and 0.5 mbar, respectively; (b) the space-charge sheath with the driven electrode (at $r=0$ and $z=4.1 \mathrm{~mm}$ ), where $\langle\varepsilon\rangle \simeq 5.9$ and $9.7 \mathrm{eV}$ for $p=1$ and 0.5 mbar, respectively. 

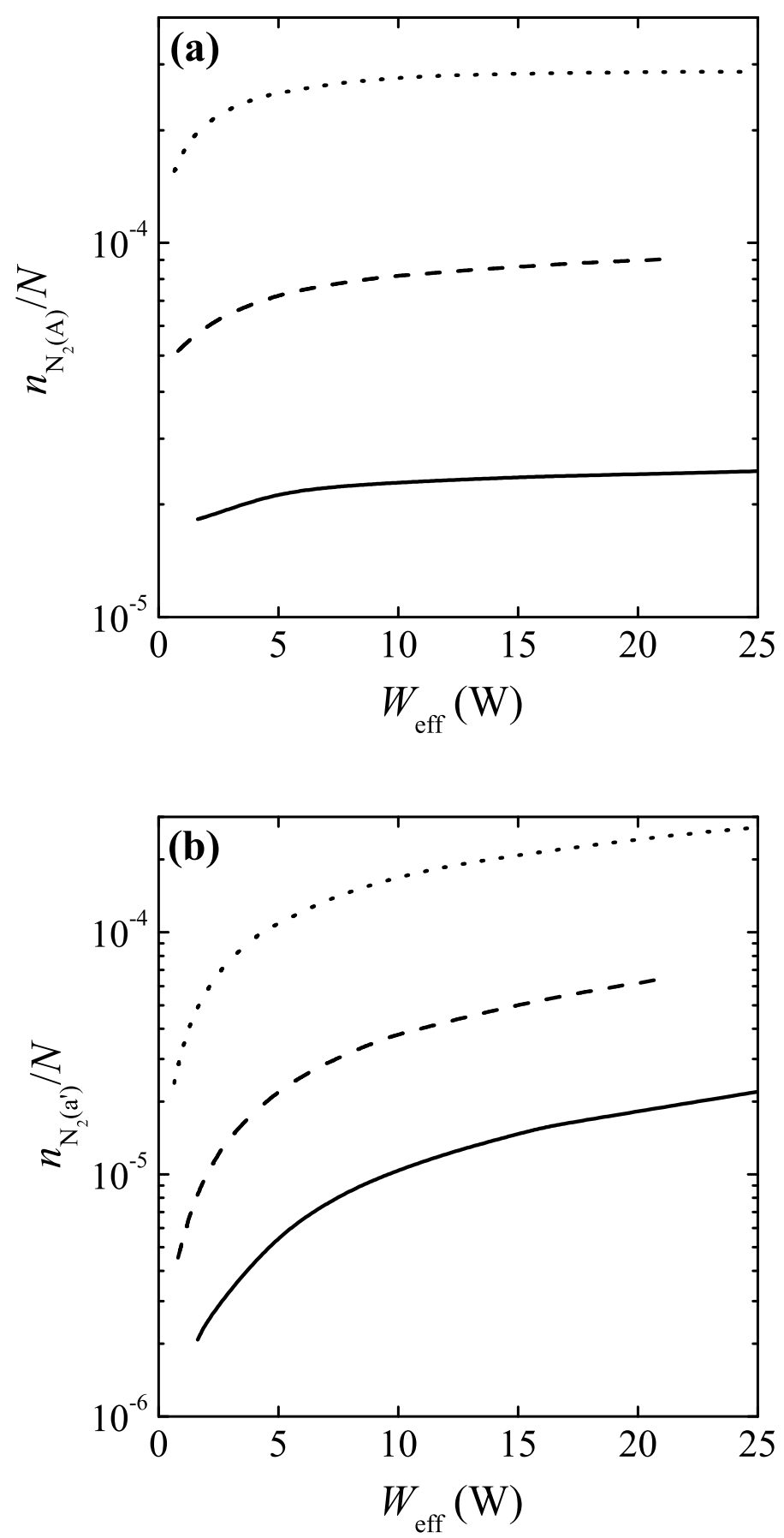

Figure 10. Reduced populations (relative to the gas density) of the $\mathrm{N}_{2}(\mathrm{~A})$ (a) and the $\mathrm{N}_{2}\left(\mathrm{a}^{\prime}\right)$ (b) species, as a function of the power coupled to the plasma, calculated for the LATMOS reactor and the following pressures (in mbar): 1 (solid line); 0.5 (dashed); 0.2 (dotted). 


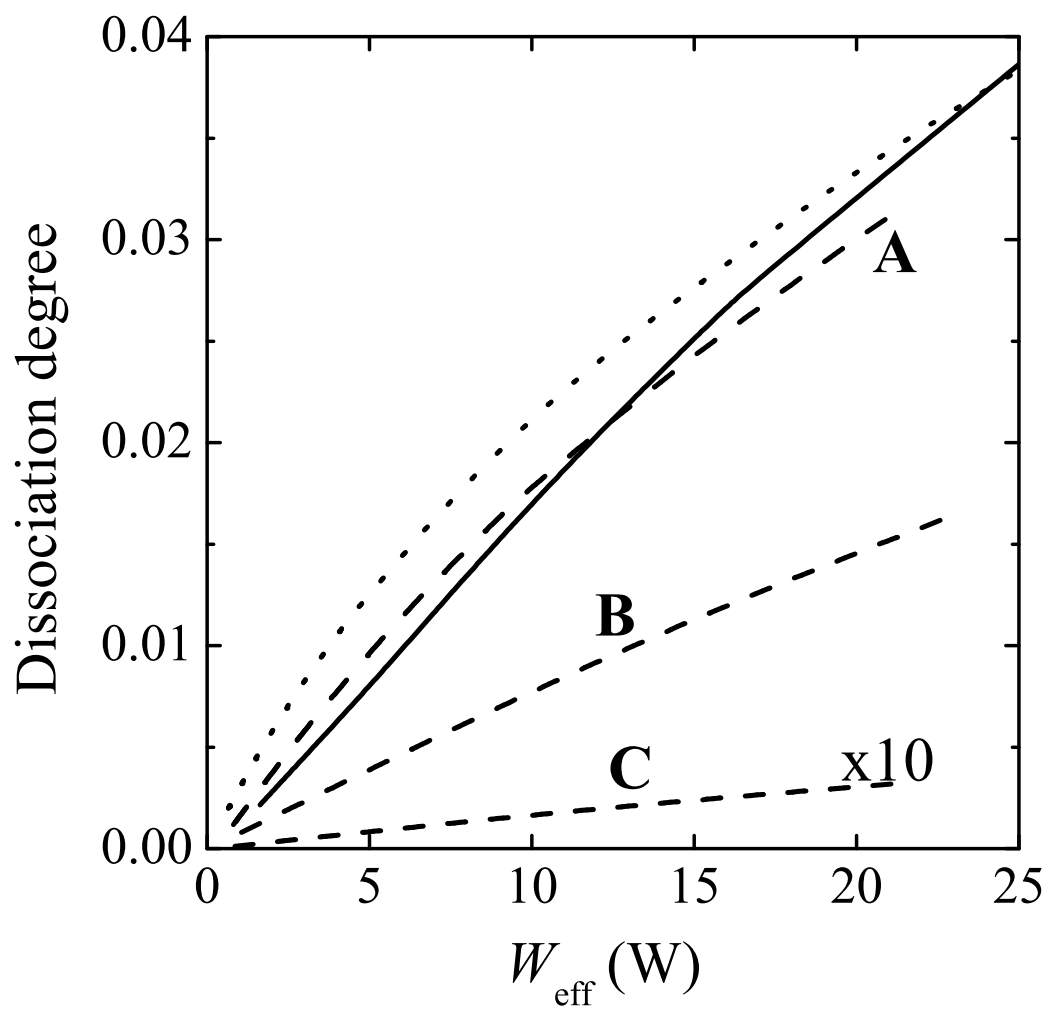

Figure 11. Dissociation degree of nitrogen $n_{\mathrm{N}} /\left(n_{\mathrm{N}_{2}}+n_{\mathrm{N}}\right)$, as a function of the power coupled to the plasma, calculated for the LATMOS reactor and the following pressures (in mbar): 1 (solid line); 0.5 (dashed); 0.2 (dotted). The dashed curves were obtained using different wall loss probabilities: $\gamma_{\mathrm{N}_{2}}^{\prime}=4.5 \times 10^{-4}$ and $\gamma_{\mathrm{N}}^{\prime}=10^{-3}$ (A, standard conditions); $\gamma_{\mathrm{N}_{2}}^{\prime}=1$ and $\gamma_{\mathrm{N}}^{\prime}$ as in A (B); $\gamma_{\mathrm{N}}^{\prime}=1$ and $\gamma_{\mathrm{N}_{2}}^{\prime}$ as in A (C, results multiplied by 10$)$. 
Capacitively coupled radio-frequency discharges in $N_{2}$

Tables and table captions 
Table 1. Nitrogen kinetic reactions for molecular species. Here, the states $\mathrm{N}_{2}\left(\mathrm{X}^{1} \Sigma_{g}^{+}, \mathrm{v}\right), \mathrm{N}_{2}\left(\mathrm{~A}^{3} \Sigma_{u}^{+}, \mathrm{B}^{3} \Pi_{g}, \mathrm{C}^{3} \Pi_{u}, \mathrm{a}^{1} \Sigma_{u}^{-}, \mathrm{a}^{1} \Pi_{g}, \mathrm{w}^{1} \Delta_{u}\right.$, $\left.\mathrm{a}^{\prime \prime 1} \Sigma_{g}^{+}\right)$and $\mathrm{N}\left({ }^{4} \mathrm{~S},{ }^{2} \mathrm{D},{ }^{2} \mathrm{P}\right)$ are represented by $\mathrm{N}_{2}(\mathrm{X}, \mathrm{v}), \mathrm{N}_{2}\left(\mathrm{~A}, \mathrm{~B}, \mathrm{C}, \mathrm{a}^{\prime}, \mathrm{a}, \mathrm{w}, \mathrm{a}^{\prime \prime}\right)$ and $\mathrm{N}(\mathrm{S}, \mathrm{D}, \mathrm{P})$, respectively; also, $\mathrm{N}_{2}(\mathrm{X})$ is for $\mathrm{N}_{2}(\mathrm{X}, \mathrm{v}=0)$, and $\mathrm{N}_{2}, \mathrm{~N}_{2}^{+}$and $\mathrm{N}$ are for the ensemble of all molecular neutral, molecular ion and atomic species, respectively. The label eedf means that the rate coefficient was obtained by integration of the cross section over the electron energy distribution function; $T_{e}$ and $T_{g}$ are the electron and the gas temperatures, respectively. The rate coefficients are in $\mathrm{cm}^{3} \mathrm{~s}^{-1}$ unless specified otherwise.

\begin{tabular}{llll}
\hline Nb. & Coll. type & Reaction & Rate coefficient \\
\hline & Electron collisions & & $C_{\alpha l, k}$ \\
\hline$(1)^{\mathrm{a}}$ & Elastic & $\mathrm{e}+\mathrm{N}_{2}(\mathrm{X}) \longrightarrow \mathrm{e}+\mathrm{N}_{2}(\mathrm{X})$ & eedf \\
$\left(2^{\mathrm{a}}\right.$ & Rot. excitation & $\mathrm{e}+\mathrm{N}_{2}(\mathrm{X}, \mathrm{J}) \longrightarrow \mathrm{e}+\mathrm{N}_{2}\left(\mathrm{X}, \mathrm{J}^{\prime}\right)$ & eedf \\
$(3)^{\mathrm{a}}$ & Vib. exc. / deexc. & $\mathrm{e}+\mathrm{N}_{2}(\mathrm{X}, \mathrm{v}=0-9) \longleftrightarrow \mathrm{e}+\mathrm{N}_{2}(\mathrm{X}, \mathrm{w}=(\mathrm{v}+1)-10)$ & eedf \\
$(4 \mathrm{a})^{\mathrm{a}}$ & Elect. exc./deexc. & $\mathrm{e}+\mathrm{N}_{2}(\mathrm{X}) \longrightarrow \mathrm{e}+\mathrm{N}_{2}\left(\mathrm{~A}, \mathrm{~B}, \mathrm{C}, \mathrm{a}^{\prime}, \mathrm{a}, \mathrm{w}, \mathrm{a}^{\prime \prime}\right)$ & eedf \\
$(4 \mathrm{~b})^{\mathrm{a}}$ & & $\mathrm{e}+\mathrm{N}_{2}(\mathrm{X}) \longrightarrow \mathrm{e}+\mathrm{N}_{2}\left(\mathrm{~B}^{\prime}\right)$ & \\
& & $\mathrm{N}_{2}\left(\mathrm{~B}^{\prime}\right) \longrightarrow \mathrm{N}_{2}(\mathrm{~B})+h \nu$ & eedf \\
$(4 \mathrm{c})^{\mathrm{b}}$ & & $\mathrm{e}+\mathrm{N}_{2}(\mathrm{X}) \longrightarrow \mathrm{e}+\mathrm{N}_{2}(\mathrm{~W}, \mathrm{E}$, higher levels $)$ & eedf \\
$(5 \mathrm{a})$ & & $\mathrm{e}+\mathrm{N}_{2}(\mathrm{~A}) \longleftrightarrow \mathrm{e}+\mathrm{N}_{2}(\mathrm{~B}, \mathrm{C})$ & eedf \\
$(5 \mathrm{~b})$ & & $\mathrm{e}+\mathrm{N}_{2}(\mathrm{~A}) \longrightarrow \mathrm{e}+\mathrm{N}_{2}(\mathrm{X})$ & eedf \\
$(6)^{\mathrm{a}}$ & Ionization & $\mathrm{e}+\mathrm{N}_{2}(\mathrm{X}) \longrightarrow 2 \mathrm{e}+\mathrm{N}_{2}^{+}(\mathrm{X}, \mathrm{B})$ & eedf \\
$(7)$ & & $\mathrm{e}+\mathrm{N}_{2}\left(\mathrm{~A}, \mathrm{~B}, \mathrm{a}^{\prime}, \mathrm{a}, \mathrm{w}\right) \longrightarrow 2 \mathrm{e}+\mathrm{N}_{2}^{+}$ & eedf \\
$(8)$ & Ion reaction & $\mathrm{e}+\mathrm{N}_{2}^{+}(\mathrm{X}) \longrightarrow \mathrm{e}+\mathrm{N}_{2}^{+}(\mathrm{B})$ & eedf \\
$(9)$ & Dissociation & $\mathrm{e}+\mathrm{N}_{2}(\mathrm{X}) \longrightarrow \mathrm{e}+\mathrm{N}_{(\mathrm{S})}+\mathrm{N}(\mathrm{S}, \mathrm{D})$ & eedf \\
$(10)$ & Recombination & $\mathrm{e}+\mathrm{N}_{2}^{+} \longrightarrow 2 \mathrm{~N}(\mathrm{~S})$ & $4.8 \times 10^{-7}\left[300 / T_{e}(\mathrm{~K})\right]^{0.5}$ \\
$(11)$ & & $\mathrm{e}+\mathrm{N}_{4}^{+} \longrightarrow 2 \mathrm{~N}_{2}(\mathrm{X})$ & $2.0 \times 10^{-6}\left[300 / T_{e}(\mathrm{~K})\right]^{0.5}$ \\
\hline
\end{tabular}


Table 1. Nitrogen kinetic reactions for molecular species (cont).

\begin{tabular}{|c|c|c|c|}
\hline $\mathrm{Nb}$. & Coll. type & Reaction & Rate coefficient \\
\hline & Heavy particle collisions & & $K_{l m, k}$ \\
\hline$(12)$ & V-T processes & $\mathrm{N}_{2}+\mathrm{N}_{2}(\mathrm{X}, \mathrm{v}=0-45) \longleftrightarrow \mathrm{N}_{2}+\mathrm{N}_{2}(\mathrm{X}, \mathrm{v} \pm 1)$ & $P_{\mathrm{v}, \mathrm{v} \pm 1}$ \\
\hline$(13)$ & $\mathrm{V}-\mathrm{V}$ processes & $\mathrm{N}_{2}(\mathrm{X}, \mathrm{v}=0-45)+\mathrm{N}_{2}(\mathrm{X}, \mathrm{w}=0-45) \longleftrightarrow \mathrm{N}_{2}(\mathrm{X}, \mathrm{v} \pm 1)+\mathrm{N}_{2}(\mathrm{X}, \mathrm{w} \mp 1)$ & $Q_{\mathrm{v}, \mathrm{v} \neq 1}^{\mathrm{w} \neq 1}$ \\
\hline$(14)$ & Vib. deexc. & $\mathrm{N}_{2}(\mathrm{~A})+\mathrm{N}_{2}(\mathrm{X}, \mathrm{v}=5-14) \longrightarrow \mathrm{N}_{2}(\mathrm{~B})+\mathrm{N}_{2}(\mathrm{X})$ & $2.0 \times 10^{-11}$ \\
\hline$(15 \mathrm{a})$ & Elect. exc./deexc. & $\mathrm{N}_{2}(\mathrm{~A})+\mathrm{N}_{2}(\mathrm{~A}) \longrightarrow \mathrm{N}_{2}(\mathrm{~B})+\mathrm{N}_{2}(\mathrm{X})$ & $7.7 \times 10^{-11}$ \\
\hline$(15 b)$ & & $\mathrm{N}_{2}(\mathrm{~A})+\mathrm{N}_{2}(\mathrm{~A}) \longrightarrow \mathrm{N}_{2}(\mathrm{C})+\mathrm{N}_{2}(\mathrm{X})$ & $1.5 \times 10^{-10}$ \\
\hline$(16 \mathrm{a})$ & & $\mathrm{N}_{2}(\mathrm{~B})+\mathrm{N}_{2} \longrightarrow \mathrm{N}_{2}(\mathrm{~A})+\mathrm{N}_{2}$ & $2.85 \times 10^{-11}$ \\
\hline$(16 b)$ & & $\mathrm{N}_{2}(\mathrm{~B})+\mathrm{N}_{2} \longrightarrow \mathrm{N}_{2}(\mathrm{X})+\mathrm{N}_{2}$ & $1.5 \times 10^{-12}$ \\
\hline$(17)$ & & $\mathrm{N}_{2}\left(\mathrm{a}^{\prime}\right)+\mathrm{N}_{2} \longrightarrow \mathrm{N}_{2}(\mathrm{~B})+\mathrm{N}_{2}$ & $1.9 \times 10^{-13}$ \\
\hline$(18)$ & & $\mathrm{N}_{2}(\mathrm{a})+\mathrm{N}_{2} \longrightarrow \mathrm{N}_{2}\left(\mathrm{a}^{\prime}\right)+\mathrm{N}_{2}$ & $2.0 \times 10^{-11}$ \\
\hline$(19)$ & & $\mathrm{N}_{2}(\mathrm{w})+\mathrm{N}_{2} \longrightarrow \mathrm{N}_{2}(\mathrm{a})+\mathrm{N}_{2}$ & $1.0 \times 10^{-11}$ \\
\hline$(20)$ & & $\mathrm{N}_{2}\left(\mathrm{a}^{\prime \prime}\right)+\mathrm{N}_{2} \longrightarrow$ products & $2.3 \times 10^{-10}$ \\
\hline$(21)$ & Dissociation & $\mathrm{N}_{2}(\mathrm{~A})+\mathrm{N}_{2}(\mathrm{X}, \mathrm{v}=14-19) \longrightarrow \mathrm{N}_{2}(\mathrm{X})+2 \mathrm{~N}(\mathrm{~S})$ & $1.5 \times 10^{-12}$ \\
\hline$(22)$ & & $2 \mathrm{~N}_{2}(\mathrm{X}, \mathrm{v}=11-24) \longrightarrow \mathrm{N}_{2}(\mathrm{X})+2 \mathrm{~N}(\mathrm{~S})$ & $3.5 \times 10^{-15}$ \\
\hline$(23)$ & Ionization & $\begin{array}{l}\mathrm{N}_{2}(\mathrm{~A})+\mathrm{N}_{2}\left(\mathrm{a}^{\prime}\right) \\
\quad \stackrel{b_{\text {ion }}}{\longrightarrow} \mathrm{N}_{4}^{+}+\mathrm{e} \\
\quad \stackrel{1-b_{\text {ipn }}}{\longrightarrow} \mathrm{N}_{2}^{+}+\mathrm{N}_{2}(\mathrm{X})+\mathrm{e}\end{array}$ & $1.0 \times 10^{-11}$ \\
\hline$(24)$ & & $\begin{aligned} \mathrm{N}_{2}\left(\mathrm{a}^{\prime}\right)+\mathrm{N}_{2}\left(\mathrm{a}^{\prime}\right) \\
\quad \stackrel{b_{\text {ion }}}{\longrightarrow} \mathrm{N}_{4}^{+}+\mathrm{e} \\
\stackrel{1-b_{\text {ipn }}}{\longrightarrow} \mathrm{N}_{2}^{+}+\mathrm{N}_{2}(\mathrm{X})+\mathrm{e}\end{aligned}$ & $5.0 \times 10^{-11}$ \\
\hline$(25)$ & Ion reactions & $\mathrm{N}_{4}^{+}+\mathrm{N}_{2} \longrightarrow \mathrm{N}_{2}^{+}+\mathrm{N}_{2}(\mathrm{X})+\mathrm{N}_{2}$ & $2.1 \times 10^{-16} \exp \left[T_{g}(\mathrm{~K}) / 121\right]$ \\
\hline$(26)$ & & $\mathrm{N}_{2}^{+}+\mathrm{N}_{2}(\mathrm{X})+\mathrm{N}_{2} \longrightarrow \mathrm{N}_{4}^{+}+\mathrm{N}_{2}$ & $6.8 \times 10^{-29}\left[300 / T_{g}(\mathrm{~K})\right]^{1.64} \mathrm{~cm}^{6} \mathrm{~s}^{-1}$ \\
\hline$(27)$ & & $\mathrm{N}_{2}^{+}(\mathrm{X})+\mathrm{N}_{2}(\mathrm{X}, \mathrm{v} \geq 12) \longrightarrow \mathrm{N}_{2}^{+}(\mathrm{B})+\mathrm{N}_{2}(\mathrm{X}, \mathrm{v}-12)$ & $1.0 \times 10^{-11}$ \\
\hline$(28)$ & Radiative trans. & $\mathrm{N}_{2}(\mathrm{~B}) \longrightarrow \mathrm{N}_{2}(\mathrm{~A})+h \nu$ & $A_{\mathrm{N}_{2}(\mathrm{~B}), \mathrm{N}_{2}(\mathrm{~A})}=2.0 \times 10^{5} \mathrm{~s}^{-1}$ \\
\hline$(29)$ & & $\mathrm{N}_{2}(\mathrm{C}) \longrightarrow \mathrm{N}_{2}(\mathrm{~B})+h \nu$ & $A_{\mathrm{N}_{2}(\mathrm{C}), \mathrm{N}_{2}(\mathrm{~B})}=2.74 \times 10^{7} \mathrm{~s}^{-1}$ \\
\hline$(30 \mathrm{a})$ & & $\mathrm{N}_{2}(\mathrm{a}) \longrightarrow \mathrm{N}_{2}(\mathrm{X})+h \nu$ & $A_{\mathrm{N}_{2}(\mathrm{a}), \mathrm{N}_{2}(\mathrm{X})}=1.8 \times 10^{4} \mathrm{~s}^{-1}$ \\
\hline$(30 b)$ & & $\mathrm{N}_{2}(\mathrm{a}) \longrightarrow \mathrm{N}_{2}\left(\mathrm{a}^{\prime}\right)+h \nu$ & $A_{\mathrm{N}_{2}(\mathrm{a}), \mathrm{N}_{2}\left(\mathrm{a}^{\prime}\right)}=1.91 \times 10^{2} \mathrm{~s}^{-1}$ \\
\hline$(31)$ & & $\mathrm{N}_{2}(\mathrm{w}) \longrightarrow \mathrm{N}_{2}(\mathrm{a})+h \nu$ & $A_{\mathrm{N}_{2}(\mathrm{w}), \mathrm{N}_{2}(\mathrm{a})}=6.5 \times 10^{2} \mathrm{~s}^{-1}$ \\
\hline$(32)$ & & $\mathrm{N}_{2}^{+}(\mathrm{B}) \longrightarrow \mathrm{N}_{2}^{+}(\mathrm{X})+h \nu$ & $A_{\mathrm{N}_{2}^{+}(\mathrm{B}), \mathrm{N}_{2}^{+}(\mathrm{X})}=1.6 \times 10^{7} \mathrm{~s}^{-1}$ \\
\hline$(33)$ & Wall reactions & $\mathrm{N}_{2}(\mathrm{X}, \mathrm{v})+$ wall $\longrightarrow \mathrm{N}_{2}(\mathrm{X}, \mathrm{v}-1)$ & $\gamma_{\mathrm{N}_{2}(\mathrm{X}, \mathrm{v})}^{\prime}$ \\
\hline$(34)$ & & $\mathrm{N}_{2}\left(\mathrm{~A}, \mathrm{a}^{\prime}, \mathrm{a}, \mathrm{w}\right) \stackrel{\text { diffusion }}{\longrightarrow} \mathrm{N}_{2}(\mathrm{X})$ & $\begin{array}{l}D_{k} N= \\
\quad 5 \times 10^{18}\left[T_{g}(\mathrm{~K}) / 300\right]^{0.5} \mathrm{~cm}^{-1} \mathrm{~s}^{-1}\end{array}$ \\
\hline
\end{tabular}

a The cross section for this mechanism is considered also in the solution to the electron Boltzmann equation.

b This mechanism is considered only in the solution to the electron Boltzmann equation. 
Table 2. Nitrogen kinetic reactions for atomic species. The labels, conventions and units are as in Table 1.

\begin{tabular}{|c|c|c|c|}
\hline Nb. & Coll. type & Reaction & Rate coefficient \\
\hline & Electron collisions & & $C_{\alpha l, k}$ \\
\hline$(35)^{\mathrm{a}}$ & Elastic & $\mathrm{e}+\mathrm{N}(\mathrm{S}) \longrightarrow \mathrm{e}+\mathrm{N}(\mathrm{S})$ & eedf \\
\hline$(36)^{\mathrm{a}}$ & Elect. exc./deexc. & $\mathrm{e}+\mathrm{N}(\mathrm{S}) \longleftrightarrow \mathrm{e}+\mathrm{N}(\mathrm{D}, \mathrm{P})$ & eedf \\
\hline \multirow[t]{2}{*}{$(37)$} & & $\mathrm{e}+\mathrm{N}(\mathrm{D}) \longleftrightarrow \mathrm{e}+\mathrm{N}(\mathrm{P})$ & eedf \\
\hline & Heavy particle collisions & & $K_{l m, k}$ \\
\hline$(38)$ & $\mathrm{V}-\mathrm{T}$ processes & $\mathrm{N}+\mathrm{N}_{2}(\mathrm{X}, \mathrm{v}=0-45) \longrightarrow \mathrm{N}+\mathrm{N}_{2}(\mathrm{X}, \mathrm{w}=0-45)$ & $P_{\mathrm{v}, \mathrm{w}}^{\mathrm{N}_{2}-\mathrm{N}}$ \\
\hline$(39)$ & Elect. exc./deexc. & $\mathrm{N}(\mathrm{D})+\mathrm{N}_{2} \longrightarrow \mathrm{N}(\mathrm{S})+\mathrm{N}_{2}$ & $1.0 \times 10^{-13} \exp \left[-510 / T_{g}(\mathrm{~K})\right]$ \\
\hline$(40)$ & & $\mathrm{N}(\mathrm{P})+\mathrm{N}_{2} \longrightarrow \mathrm{N}(\mathrm{S})+\mathrm{N}_{2}$ & $6.0 \times 10^{-14}$ \\
\hline$(41)$ & & $\mathrm{N}(\mathrm{P})+\mathrm{N}_{2}(\mathrm{X}, \mathrm{v} \geq 10) \longrightarrow \mathrm{N}(\mathrm{S})+\mathrm{N}_{2}(\mathrm{~A})$ & $1.0 \times 10^{-10} \exp \left[-1300 / T_{g}(\mathrm{~K})\right]$ \\
\hline$(42)$ & & $\mathrm{N}(\mathrm{S})+\mathrm{N}_{2}(\mathrm{~A}) \longrightarrow \mathrm{N}(\mathrm{P})+\mathrm{N}_{2}(\mathrm{X}, \mathrm{v}=6-9)$ & $4.0 \times 10^{-11}$ \\
\hline$(43 a)$ & & $\mathrm{N}(\mathrm{S})+\mathrm{N}(\mathrm{P}) \longrightarrow \mathrm{N}(\mathrm{S})+\mathrm{N}(\mathrm{S})$ & $1.8 \times 10^{-12}$ \\
\hline$(43 b)$ & & $\mathrm{N}(\mathrm{S})+\mathrm{N}(\mathrm{P}) \longrightarrow \mathrm{N}(\mathrm{S})+\mathrm{N}(\mathrm{D})$ & $6.0 \times 10^{-13}$ \\
\hline$(44)$ & & $\mathrm{N}(\mathrm{S})+\mathrm{N}(\mathrm{S})+\mathrm{N}_{2} \longrightarrow \mathrm{N}_{2}(\mathrm{~B})+\mathrm{N}_{2}$ & $8.27 \times 10^{-34} \exp \left[500 / T_{g}(\mathrm{~K})\right] \mathrm{cm}^{6} \mathrm{~s}^{-1}$ \\
\hline$(45)$ & Ionization & $\mathrm{N}(\mathrm{D})+\mathrm{N}(\mathrm{P}) \longrightarrow \mathrm{e}+\mathrm{N}_{2}^{+}$ & $1.0 \times 10^{-13}$ \\
\hline$(46)$ & Wall reactions & $\mathrm{N}(\mathrm{S})+$ wall $\longrightarrow(1 / 2) \mathrm{N}_{2}(\mathrm{X})$ & $\gamma_{\mathrm{N}(\mathrm{S})}^{\prime}$ \\
\hline \multirow[t]{3}{*}{$(47)$} & & $\mathrm{N}(\mathrm{D}) \stackrel{\text { diffusion }}{\longrightarrow}$ & $D_{\mathrm{N}(\mathrm{D})} N=6.4 \times 10^{18}\left[T_{g}(\mathrm{~K}) / 300\right]^{0.5} \mathrm{~cm}^{-1} \mathrm{~s}^{-1}$ \\
\hline & & $\stackrel{b_{\text {atom }}}{\longrightarrow} \mathrm{N}(\mathrm{S})$ & \\
\hline & & $\stackrel{1-b_{\text {atom }}}{\longrightarrow}(1 / 2) \mathrm{N}_{2}(\mathrm{X})$ & \\
\hline \multirow[t]{3}{*}{$(48)$} & & $\mathrm{N}(\mathrm{P}) \stackrel{\text { diffusion }}{\longrightarrow}$ & $D_{\mathrm{N}(\mathrm{P})} N=5.2 \times 10^{18}\left[T_{g}(\mathrm{~K}) / 300\right]^{0.5} \mathrm{~cm}^{-1} \mathrm{~s}^{-1}$ \\
\hline & & $\stackrel{b_{\text {atom }}}{\longrightarrow} \mathrm{N}(\mathrm{S})$ & \\
\hline & & $\stackrel{1-b_{\text {atom }}}{\longrightarrow}(1 / 2) \mathrm{N}_{2}(\mathrm{X})$ & \\
\hline
\end{tabular}

a The cross section for this mechanism is considered also in the solution to the electron Boltzmann equation. 
Table 3. Quantities to be used in equation (26a), of the rate coefficient for V-T collisions with atoms. $A_{0}$ is in $\mathrm{cm}^{3} \mathrm{~s}^{-1}$ and $T_{g}$ is in $\mathrm{K}$.

\begin{tabular}{lll}
\hline & Reactive & Non-reactive \\
\hline$A_{0}$ & $2.21 \times 10^{4} / T_{g}^{1.43}$ & $9.24 \times 10^{4} / T_{g}^{1.63}$ \\
$A_{1}$ & $3.21 \times 10^{4} / T_{g}^{0.80}$ & $1.82 \times 10^{4} / T_{g}^{0.70}$ \\
$A_{2}$ & $2.50 \times 10^{5} / T_{g}^{1.04}$ & $9.89 \times 10^{3} / T_{g}^{0.44}$ \\
$\mathrm{v}^{\star}$ & 7 & 9 \\
\hline
\end{tabular}




\section{References}

[1] Ata M, Huang H and Akasaka T 2004 J. Phys. Chem. B. 1084640

[2] Miyanaga S, Kaneko T, Ishida H and Hatakeyama R 2010 Thin Solid Films 5183509

[3] Brüser V, Heintze M, Brandl W, Marginean G and Bubert H 2004 Diamond and Related Materials 131177

[4] Abbas G, Papakonstantinou P, Iyer G R S, Kirkman I W and Chen L C 2007 Phys. Rev. B 75 195429

[5] Bertóti I, Mohai I, Mohai M and Szépvölgyi J 2011 Diamond and Related Materials 20965

[6] Yang C, Lee S, Wu J and Lin 2005 Appl. Surf. Sci. 2521818

[7] Vesel A, Mozetic M and Zalar A 2008 Surf. Interface Anal. 40661

[8] Zhang S, Awaja F, James N, McKenzie D R and Ruys A J 2011 Polym. Adv. Technol. 222496

[9] Morent R, De Geyter N, Desmet T, Dubruel P and Leys C 2011 Plasma Process. Polym. 8171

[10] Hossain M M, Herrmann A S and Hegemann D 2007 Plasma Process. Polym. 4135

[11] Vesel A, Junkar I, Cvelbar U, Kovac J and Mozetic M 2008 Surf. Interface Anal. 401444

[12] Kikuchi T, Somintac A S, Ariyada O, Wada M and Ohachi T 2006 Journal of Crystal Growth 292221

[13] Kesaria M, Shetty S and Shivaprasad S M 2011 Journal of Crystal Growth 326191

[14] Ramsey M E, Poindexter E, Pelt J S, Marin J and Durbin S M 2000 Thin Solid Films 36082

[15] Bhattacharyya S, Auciello O, Birrell J, Carlisle J A, Curtiss L A, Goyette A N, Gruen D M, Krauss A R, Schlueter J, Sumant A and Zapol P 2001 Appl. Phys. Lett. 791441

[16] Hayashi Y, Krishna K M, Ebisu H, Soga T, Umeno M and Jimbo T 2001 Diamond and Related Materials 101002

[17] Sachdev H and Scheid P 2001 Diamond and Related Materials 101160

[18] Anita V, Butuda T, Maeda T, Takizawa K, Saito N, Takai O 2004 Diamond and Related Materials 131993

[19] Miyajima Y, Henley S J and Silva S R P 2011 Thin Solid Films 5196374

[20] Othman M, Ritikos R, Khanis N H, Rashid N M A, Rahman S A, Gani S M A and Muhamad M R 2011 Thin Solid Films $\mathbf{5 1 9} 4981$

[21] Tzeng S-S, Fang Y-L, Chih Y-K, Hu Y-G, Hsu J-S, Wu C-L and Wu G-J 2011 Thin Solid Films 5194870

[22] Kovačević E, Berndt J, Stefanović I, Becker H-W, Godde C, Strunskus Th, Winter J and Boufendi L 2009 J. Appl. Phys. 105104910

[23] Kovačević E, Stefanović I, Berndt J, Pendleton Y J and Winter J 2005 Astrophys. J. 623242

[24] Franceschini D F, Achete C A and Freire F L 1992 Appl. Phys. Lett. 603229

[25] Siow K S, Britcher L, Kumar S and Griesser H J 2006 Plasma Process. Polym. 3392

[26] Truica-Marasescu F and Wertheimer M R 2008 Plasma Process. Polym. 544

[27] Petit A, Wang H T, Girard-Lauriault P-L, Antoniou J, Wertheimer M R and Mwale F $2010 \mathrm{~J}$. Biomed. Mater. Res.: Part A 94a 744

[28] Mwale F, Wang H T, Petit A, Girard-Lauriault P-L, Wertheimer M R and Antoniou J 2008 J. Stem. Cells. 387

[29] Szopa C, Cernogora G, Boufendi L, Correia J J and Coll P 2006 Planet. Space Sci. 54394

[30] Khare B N, Sagan C, Zumberge J E, Sklarew D S and Nagy B 1981 Icarus 48290

[31] Sagan C, Thompson W R and Khare B N 1992 Acc. Chem. Res. 25286

[32] Coll P, Coscia D, Smith N, Gazeau M-C, Ramirez S I, Cernogora G, Isral G and Raulin F 1999 Planet. Space Sci. $\mathbf{4 7} 1331$

[33] Pintassilgo C D, Cernogora G and Loureiro J 1999 Plasma Sources Sci. Technol. 8463

[34] Bernard J M, Coll P, Coustenis A and Raulin F 2003 Planet. Space Sci. 511003

[35] Fujii T and Arai N 1999 Astrophys. J. $\mathbf{5 1 9} 858$

[36] Es-sebbar Et, Gazeau M-C, Benilan Y and Jolly A 2009 J. Phys. D: Appl. Phys. 43335203

[37] Imanaka H, Khare B N, Elsila J E, Bakes E L O, McKay C P, Cruikshank D P, Sugita S, Matsui 
T and Zare R N 2004 Icarus 168344

[38] Ramirez S I, Navarro-Gonzalez R, Coll P and Raulin F 2005 Adv. Space Res. 36274

[39] Horvath G, Skalny J D, Mason N J, Klas M, Zahoran M, Vladoiu R and Manole M 2009 Plasma Sources Sci. Technol. 18034016

[40] Horvath G, Mason N J, Polachova L, Zahoran M, Moravsky L and Matejcik S 2010 Plasma Chem. Plasma Process. 30565

[41] Alcouffe G, Cavarroc M, Cernogora G, Ouni F, Jolly A, Boufendi L and Szopa C 2010 Plasma Sources Sci. Technol. 19015008

[42] Pereira J, Géraud-Grenier I, Massereau-Guilbaud V, Plain A 2005 Thin Solid Films 482226

[43] Pereira J, Géraud-Grenier I, Massereau-Guilbaud V, Plain A and Fernandez V 2006 Surface and Coatings Technology 2006414

[44] Cernogora G, Ferreira C M, Hochard L, Touzeau M and Loureiro J 1984 J. Phys. B: At. Mol. Phys. 174429

[45] Ferreira C M, Touzeau M, Hochard L and Cernogora G 1984 J. Phys. B: At. Mol. Phys. 174439

[46] Loureiro J and Ferreira C M 1986 J. Phys. D: Appl. Phys. 1917

[47] Loureiro J and Ferreira C M 1989 J. Phys. D: Appl. Phys. 2267

[48] Magne L, Cernogora G, Loureiro J and Ferreira C M 1991 J. Phys. D: Appl. Phys. 241758

[49] Guerra V and Loureiro J 1997 Plasma Sources Sci. Technol. 6361

[50] Petrusev A S, Surzhikov S T and Shang J S 2006 High Temperature 44804 (Translated from 2006 Teplofizika Vysokikh Temperatur 44 814)

[51] Tatarova E, Dias F M, Ferreira C M, Guerra V, Loureiro J, Stoykova E, Ghanashev I and Zhelyazkov I 1997 J. Phys. D: Appl. Phys. 302663

[52] Tatarova E, Dias F M, Ferreira C M and Ricard A 1999 J. Appl. Phys. 8549

[53] Guerra V, Tatarova E, Dias F M and Ferreira C M 2002 J. Appl. Phys. 912648

[54] Douai D, Berndt J and Winter J 2002 Plasma Sources Sci. Technol. 1160

[55] Ferreira C M, Tatarova E, Guerra V, Gordiets B, Henriques J, Dias F M and Pinheiro M 2003 IEEE Trans. Plasma Sci. 31645

[56] Hong S, Douai D, Berndt J and Winter J 2005 Plasma Sources Sci. Technol. 14451

[57] Thorsteinsson E G and Gudmundsson J T 2009 Plasma Sources Sci. Technol. 18045001

[58] Kushner M J 1983 J. Appl. Phys. 544958

[59] Longo S and Capitelli M 1994 Phys. Rev. E 492302

[60] Sommerer T J and Kushner M J 1992 J. Appl. Phys. 711654

[61] Longo S, Hassouni K, Iasillo D and Capitelli M 1997 J. Phys. III France 7707

[62] Turner M M and Hopkins M B 1992 Phys. Rev. Lett. 693511

[63] Lieberman M A and Lichtenberg A J 2005 Principles of Plasma Discharges and Materials Processing (New York: Wiley) pp. 32, 37, 67

[64] Raiser Y P 1991 Gas Discharge Physics (Berlin: Springer) p. 37

[65] Salabas A, Gousset G and Alves L L 2002 Plasma Sources Sci. Technol. 11448

[66] Alves L L 2007 Plasma Sources Sci. Technol. 16557

[67] Godyak V A and Piejak R B 1990 J. Vac. Sci. Technol A 83883

[68] Horwitz C M 1983 J. Vac. Sci. Technol. A 11795

[69] Howling A A, Hollenstein Ch, Paris P J, Finger F and Kroll U, 1991 XX International Conference on Phenomena in Ionized Gases Vol 5, 1089

[70] Papoular R and Balazard J 1965 Applications des ondes hyperfréquences et infrarouges à l'étude des plasmas (Paris: Dunod)

[71] Brown S C and Ingraham J C 1966 Plasma diagnostics - technical report 454 (Massachusetts Institute of Technology - Research Laboratory of Electronics)

[72] Haverlag M, Kroesen G M W, Bisschops T H J and De Hoog F J 1991 Plasma Chem. Plasma Process. 11357

[73] Boufendi L, Bouchoule A and Hbid T 1996 J. Vac. Sci. Technol A 14572

[74] Lofthus A and Krupenie P H 1977 J. Phys. Chem. Ref. Data 6113 
[75] Salabas A, Marques L, Jolly J, Gousset G and Alves L L 2004 J. Appl. Phys. 954605

[76] Marques L, Jolly J and Alves L L 2007 J. Appl. Phys. 102, 063305

[77] Richards A D, Thompson B E and Sawin H H 1987 Appl. Phys. Lett. 50492

[78] Ellis H W, Pai R Y, McDaniel E W, Mason E A and Viehland L A 1976 At. Data Nucl. Data Tables $\mathbf{1 7} 177$

[79] Phelps A V 1991 J. Phys. Chem. Ref. Data 20557

[80] Guerra V, Sá P A and Loureiro J 2004 Eur. Phys. J. Appl. Phys. 28125

[81] Davison B and Sykes J B 1958 Neutron Transport Theory (Oxford: Clarendon)

[82] Chantry P J 1987 J. Appl. Phys. 621141

[83] Phelps A V 1985 Technical Report 28 (JILA Information Center Report, University of Colorado, Boulder, Colorado, USA)

[84] Klein O and Rosseland S 1921 Z. Phyz. 446

[85] Schwartz R N, Slawsky Z I and Herzfeld K F 1952 J. Chem. Phys. 201591

[86] Schwartz R N and Herzfeld K F 1954 J. Chem. Phys. 22767

[87] Keck J and Carrier G 1965 J. Chem. Phys. 432284

[88] Bray K N C 1968 J. Phys. B: At. Mol. Phys. 1705

[89] Billing G D and Fisher E R 1979 Chem. Phys. 43395

[90] Frost R J and Smith I W M 1987 Chem. Phys. Lett. 140499

[91] Armenise I, Capitelli M, Garcia E, Gorse C, Laganà A and Longo S 1992 Chem. Phys. Lett. 200 597

[92] Laganà A, Ochoa de Aspuru G, Garcia E 1996 Quasiclassical and quantum rate coefficients for the $\mathrm{N}+\mathrm{N}_{2}$ reaction, Technical report (Università degli Studi di Perugia)

[93] Garcia E and Laganà A 1997 J. Phys. Chem. 1014734

[94] Capitelli M and Dilonardo M 1977 Chem. Phys. 24417

[95] Scharfetter D L and Gummel H-K 1969 IEEE Trans. Electron Devices 1664

[96] Courant R, Friedrichs K and Lewy H 1967 IBM Journal of Research and Development 11215

[97] Hancock G, Peverall R, Ritchie G A D and Thornton L J 2006 J. Phys. D: Appl. Phys. 391846 